\title{
In-operation field-of-view retrieval (IFR) for satellite and ground-based DOAS-type instruments applying coincident high-resolution imager data
}

\author{
Holger Sihler ${ }^{1,2}$, Peter Lübcke ${ }^{2}$, Rüdiger Lang ${ }^{3}$, Steffen Beirle ${ }^{1}$, Martin de Graaf ${ }^{4,5}$, Christoph Hörmann ${ }^{1}$, \\ Johannes Lampel ${ }^{1}$, Marloes Penning de Vries ${ }^{1}$, Julia Remmers ${ }^{1}$, Ed Trollope ${ }^{3,6}$, Yang Wang ${ }^{1}$, and Thomas Wagner ${ }^{1}$ \\ ${ }^{1}$ Max Planck Institute for Chemistry (MPIC), Hahn-Meitner-Weg 1, 55128 Mainz, Germany \\ ${ }^{2}$ Institute of Environmental Physics (IUP), University of Heidelberg, Im Neuenheimer Feld 229, 69120 Heidelberg, Germany \\ ${ }^{3}$ European Organisation for the Exploitation of Meteorological Satellites (EUMETSAT), Eumetsat Allee 1, 64295 Darmstadt, \\ Germany \\ ${ }^{4}$ Royal Netherlands Meteorological Institute (KNMI), Utrechtseweg 297, 3731 GA De Bilt, the Netherlands \\ ${ }^{5}$ Delft University of Technology (TU-Delft), Stevinweg 1, 2628 CN Delft, the Netherlands \\ ${ }^{6}$ Telespazio VEGA Deutschland GmbH, Europaplatz 5, 64293 Darmstadt, Germany
}

Correspondence to: Holger Sihler (holger.sihler@mpic.de)

Received: 29 June 2016 - Discussion started: 17 August 2016

Revised: 1 February 2017 - Accepted: 1 February 2017 - Published: 9 March 2017

\begin{abstract}
Knowledge of the field of view (FOV) of a remote sensing instrument is particularly important when interpreting their data and merging them with other spatially referenced data. Especially for instruments in space, information on the actual FOV, which may change during operation, may be difficult to obtain. Also, the FOV of ground-based devices may change during transportation to the field site, where appropriate equipment for the FOV determination may be unavailable.

This paper presents an independent, simple and robust method to retrieve the FOV of an instrument during operation, i.e. the two-dimensional sensitivity distribution, sampled on a discrete grid. The method relies on correlated measurements featuring a significantly higher spatial resolution, e.g. by an imaging instrument accompanying a spectrometer. The method was applied to two satellite instruments, GOME-2 and OMI, and a ground-based differential optical absorption spectroscopy (DOAS) instrument integrated in an $\mathrm{SO}_{2}$ camera. For GOME-2, quadrangular FOVs could be retrieved, which almost perfectly match the provided FOV edges after applying a correction for spatial aliasing inherent to GOME-type instruments. More complex sensitivity distributions were found at certain scanner angles, which are probably caused by degradation of the moving parts within
\end{abstract}

the instrument. For OMI, which does not feature any moving parts, retrieved sensitivity distributions were much smoother compared to GOME-2. A 2-D super-Gaussian with six parameters was found to be an appropriate model to describe the retrieved OMI FOV. The comparison with operationally provided FOV dimensions revealed small differences, which could be mostly explained by the limitations of our IFR implementation. For the ground-based DOAS instrument, the FOV retrieved using $\mathrm{SO}_{2}$-camera data was slightly smaller than the flat-disc distribution, which is assumed by the stateof-the-art correlation technique. Differences between both methods may be attributed to spatial inhomogeneities.

In general, our results confirm the already deduced FOV distributions of OMI, GOME-2, and the ground-based DOAS. It is certainly applicable for degradation monitoring and verification exercises. For satellite instruments, the gained information is expected to increase the accuracy of combined products, where measurements of different instruments are integrated, e.g. mapping of high-resolution cloud information, incorporation of surface climatologies. For the $\mathrm{SO}_{2}$-camera community, the method presents a new and efficient tool to monitor the DOAS FOV in the field. 


\section{Introduction}

The instantaneous field of view (IFOV) of an optical instrument describes the solid angle from which radiation is perceived by a detector. If the instrument is moving, averaging the IFOV over the integration time of one measurement yields the field of view (FOV). The term FOV used here refers to the spatial sensitivity distribution of the acquisition method rather than the distinct transfer properties of a point source through an optical system, which are usually referenced as point spread function (PSF), spatial transfer function, modulation transfer function (MTF), or impulse response of a system. In practice, the FOV is often assumed quadrangular or elliptic with a constant sensitivity inside and zero sensitivity outside. This study demonstrates that this is a strong simplification and that some spectroscopic instruments feature a more complex FOV.

For satellite measurements, the extent and shape of the FOV are of particular interest in order to register the measured quantity in space. Accurate data registration is an important prerequisite for further data processing and comparison with other georeferenced data. In principle, a priori information on the IFOV is available from measurements in a controlled environment or raytracing simulations, e.g. OMI (te Plate et al., 2001; Dobber et al., 2006), MODIS (Xiong et al., 2005), GOME-2B (EUMETSAT, 2011b), and VIIRS (Wolfe et al., 2013). The FOV then follows from geometric considerations. The alignment of the optical components, however, may change when deploying a satellite instrument in orbit or a ground-based instrument in the field. Hence, it is desirable to infer the actual FOV at any time.

One possibility to obtain the FOV from measurements by the instrument itself is to scan over well-known edges and structures. For example, several methods to infer the FOV of imaging satellite instruments during operation (i.e. in orbit) take advantage of man-made structures. McGillem et al. (1983) retrieved the PSF of Landsat imagery using field edges, Ruiz and Lopez (2002) derived the PSF by applying deconvolution filters to images of a large dam, and Campagnolo and Montaño (2014) exploited the linearity of dikes in the Netherlands. Another approach demonstrated by Wang et al. (2014) derived the MTF of MODIS from scans of the lunar disc. For ground-based MAX-DOAS (Platt and Stutz, 2008), the vertical FOV shape and position are sometimes inferred from horizon scans provided that the horizon is visible and features a sufficiently strong radiometric gradient.

Spectrometers divide the electromagnetic spectrum into a much higher number of spectral channels (e.g. GOME-2: 4096) compared to imagers (e.g. MODIS: 36). The bandwidth of spectrometers is much smaller, and, hence, spectroscopic measurements need a significantly larger integration time than imagers in order to achieve a similar signal-tonoise ratio (SNR) due to photon statistics. The spatial resolution of spectroscopic instruments on satellites is therefore usually too coarse to use man-made structures or the Moon for FOV retrievals.

For spectroscopic satellite retrievals, the FOV edges are assumed sharp in most applications (e.g. Koelemeijer et al., 1998; Kroon et al., 2008). This may be reasonable for scanning instruments like GOME, SCIAMACHY, and GOME2, but imaging spectrometers, like OMI, have a more complex, approximately bell-shaped FOV (Kurosu and Celarier, 2010). To address these peculiarities, Kuhlmann et al. (2014) developed an interpolation scheme for OMI data based on parabolic spline surfaces, and Siddans (2016) proposed an approach to map high-resolution cloud data on TROPOMI measurements applying spectrally resolved FOVs obtained during pre-launch instrument calibration (Leloux, 2016). Furthermore, de Graaf et al. (2016) obtained the parameters of a 2-D super-Gaussian FOV by searching for maximum correlation between OMI and MODIS measurements (differences to this study are discussed in Sect. 4.2).

For passive ground-based DOAS instruments using scattered solar light, the FOV is often characterized only in the vertical direction using artificial line light sources, which is a sufficient approach for common multi-axis DOAS applications when the measurement signal is almost constant in the lateral direction. This simplification, however, may become inappropriate when the DOAS instrument is used in combination with an imaging instrument, such as an $\mathrm{SO}_{2}$ camera (Mori and Burton, 2006; Bluth et al., 2007; Kern et al., 2010). Built-in DOAS instruments are increasingly used to calibrate $\mathrm{SO}_{2}$-camera images, because DOAS measures $\mathrm{SO}_{2}$ column densities more accurately due to the the technique's robustness with respect to interferences with other absorbers in the light path. The calibration procedure requires $\mathrm{SO}_{2} \mathrm{col}-$ umn density measurements from both instruments accurately matched in space and time, i.e. the correlation between both measurements must be very high. In order to maximize the correlation, accurate knowledge of the FOV of the spectrometer within the $\mathrm{SO}_{2}$-camera image is necessary. In the past, the FOV was often found by assuming a certain shape for the FOV - mostly a disc of varying diameter - and calculating the correlation between the optical density and the $\mathrm{SO}_{2}$ column density depending on disc diameter (e.g. Kern et al., 2010; Lübcke et al., 2013). However, this method is computationally expensive and an irregular shape of the spectrometer FOV can distort the results.

In this paper, we propose a method to retrieve discretized FOVs of spatially low-resolving (LR) spectrometers from correlated high-resolution (HR) measurements. The in-operation FOV retrieval (IFR) method relies on a sufficiently large set of $m$ inhomogeneous HR measurements, which need to be spatially aligned to the corresponding LR measurements. Three exemplary LR/HR instrument combinations are investigated to demonstrate the applicability to both satellite and ground-based instruments: (1) GOME2/AVHRR, (2) OMI/MODIS, and (3) passive DOAS/ $/ \mathrm{SO}_{2}$ camera. 
The paper is organized as follows. Details of the instruments and data sets of these LR/HR combinations are provided in Sect. 2.1. Section 2.2 describes the spatial resampling of the HR measurements, and the formal approach of IFR is explained in Sect. 2.3. Furthermore, Sect. 2.4 proposes a 2-D FOV parametrization. The resulting FOV are presented in Sect. 3, and discussed in Sect. 4, for the considered LR/HR pairs, followed by conclusions. Retrieval errors for the GOME-2 results are estimated in Appendix A.

\section{Methods}

\subsection{Input data}

\subsubsection{GOME-2/AVHRR}

The first LR instrument, whose FOVs are investigated, is the second-generation Global Ozone Monitoring Experiment (GOME-2, Callies et al., 2000; Munro et al., 2006, 2016). GOME-2 is one of several instruments on the MetOp satellite. Two of the three essentially identical MetOp satellites are in orbit: MetOp-A and MetOp-B, which were launched in 2006 and in 2012, respectively. This study is limited to the GOME-2 instrument on MetOp-A.

GOME-2 features four spectroscopic main channels (science channels) between 240 and $790 \mathrm{~nm}$ with a spectral resolution between 0.26 and $0.51 \mathrm{~nm}$. Furthermore, GOME2 includes two polarization measurement devices (PMDs) whose measurements are clustered to 15 PMD channels each (Lang, 2010; Tilstra et al., 2011). The instrument features a maximum swath width of $1920 \mathrm{~km}$ scanned applying the whisk-broom approach as depicted in Fig. 1. In July 2013, however, the nominal swath width of GOME-2 on MetOpA was changed to $960 \mathrm{~km}$. The IFOV in across-track and along-track direction are 4 and $40 \mathrm{~km}$, respectively (Munro et al., 2016). One scan of GOME- 2 consists of a $4.5 \mathrm{~s}$ forward scan and a $1.5 \mathrm{~s}$ backward scan divided into 24 and 8 pixels, respectively. Hence, the nominal pixel sizes of the four main science channels (MSCs) was $80 \mathrm{~km} \times 40 \mathrm{~km}$ before July 2013. The PMD readouts are performed at an 8 times higher rate, resulting in 256 PMD pixels per scan and leading to a nominal pixel size of $10 \mathrm{~km} \times 40 \mathrm{~km}$ for a forward-scan PMD pixel. It is noted that GOME-2 features a variable speed of the scanner motor in order to compensate for Earth curvature and to maintain a regular pixel size in the across-track direction. Furthermore, until June 2013, the swath width of GOME- 2 was reduced to $240 \mathrm{~km}$ (narrow mode) every 29 days providing an improved nominal resolution of $10 \mathrm{~km} \times 40 \mathrm{~km}$ for the MSCs.

EUMETSAT provides coordinates representing the corners of a rectangular FOV for each GOME-2 measurement, i.e. one GOME-2 pixel, which is calculated from the readout timing and the scanner position. The FOV is typically assumed constant inside and zero outside the provided edges,

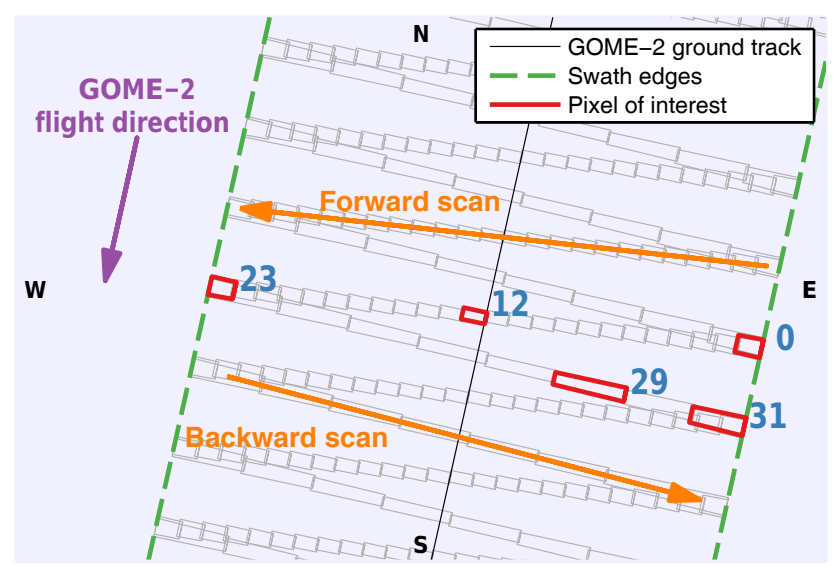

Figure 1. Schematic of GOME-2 whisk-broom scanning regime consisting of a $4.5 \mathrm{~s}$ forward scan and a $1.5 \mathrm{~s}$ backward scan. Note that the along-track displacement (along the flight direction) is enhanced by a factor of 7 between the pixels for the sake of clarity in reality, there are no gaps between consecutive forward scans. The highlighted pixels are studied in more detail.

respectively. The actual FOV edges, however, need to be shifted relative to the provided FOV geolocations depending on wavelength since the detector pixels are not read out simultaneously. This shift caused by the mirror movement during readout leads to the spatial aliasing effect (EUMETSAT, 2015c; Munro et al., 2016), whose influence on the radiometric correlation between HR and LR measurements has already been discussed by Koelemeijer et al. (1998) for the first GOME instrument. The relative shift towards the subsequent FOV can be calculated from the detector pixel number for each detector (four MSC and two PMD detectors) separately. The detector pixel to wavelength mapping is provided in EUMETSAT (2015c) and Munro et al. (2016). Effectively, the spatial aliasing for the MSCs is between zero and $26 \%$ relative to the position of the succeeding FOV. The corresponding spatial offset of a nominal $80 \mathrm{~km} \times 40 \mathrm{~km}$ pixel is between 0 and $21 \mathrm{~km}$ in across-track direction.

The FOV of all nominal 32 MSC pixels were retrieved, but both narrow-mode FOV and PMD FOV were only retrieved in the nadir direction. At nadir, neighbouring FOVs are very similar, and therefore the accuracy could be improved by combining neighbouring pixels of the same scan for narrowmode and PMD FOVs. The four neighbouring pixels east and west of the nadir pixel were included in the FOV retrieval of the same nadir pixel for a 9-fold number of measurements $m$. This approach was particularly useful to reduce the time period required to retrieve the narrow-mode FOV because the narrow mode is activated only every 29th day (see below).

In this study, the GOME-2 FOVs were retrieved from the combination with measurements by the AVHRR/3 (Advanced Very High Resolution Radiometer) instrument also installed on the MetOP-A satellite (Cracknell, 1997; NOAA, 2009; EUMETSAT, 2011a). AVHRR features a nadir reso- 


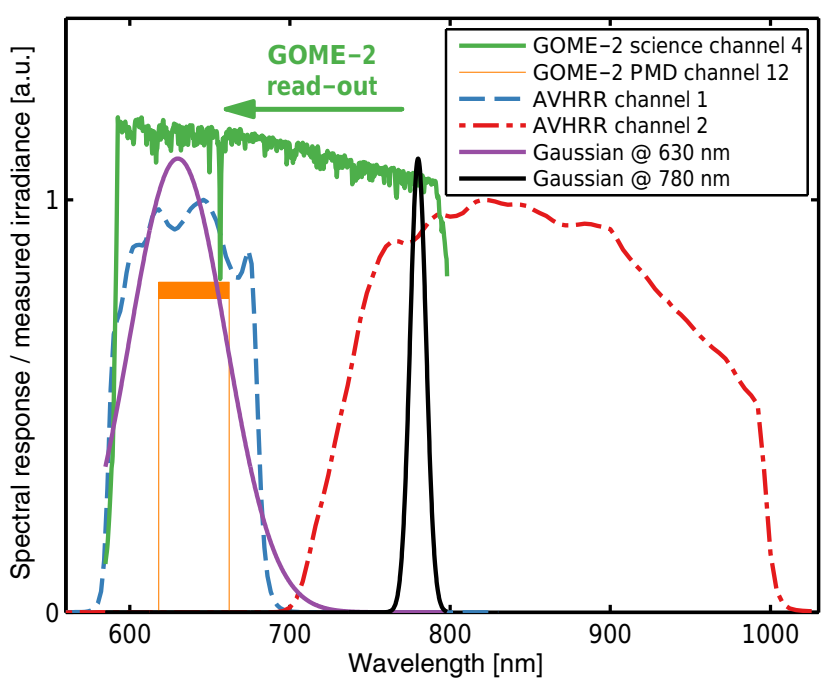

Figure 2. Solar irradiance spectrum measured by science channel 4 of GOME-2 on 1 April 2009, the spectral range covered by GOME2 PMD channel 12, and the sensitivity response of AVHRR/MetOp channels 1 and 2 (Lang, 2010; NOAA, 2014). In addition, two Gaussians used to convolve GOME-2 spectra are shown.

lution of $1.1 \mathrm{~km}$ and acquires data in five spectral channels between the visible red and thermal infrared (NOAA, 2014). The first two channels of AVHRR are used in this study because only these both overlap with the spectral range covered by GOME-2 as illustrated in Fig. 2. The spectral response of AVHRR channel 1 centred at $630 \mathrm{~nm}$ is almost entirely located within GOME-2 science channel 4 at its left edge. The spectral response of AVHRR channel 2, however, exceeds that of GOME- 2 channel 4 towards the infrared.

Five different spectral convolution kernels were applied to MSC 4 data of GOME-2 in order to investigate the correlation between GOME-2 and AVHRR radiances and the tradeoff between minimizing spatial aliasing and maximizing spectral overlap between GOME-2 and AVHRR: the spectral response of AVHRR channels 1 and 2 (NOAA, 2014), the spectral response of PMD channel 12 approximated by a box profile between 618 and $662 \mathrm{~nm}$ (Lang, 2010), one Gaussian centred at 630 and $30 \mathrm{~nm}$ width, and one Gaussian at 780 and $5 \mathrm{~nm}$ width (depicted in Fig. 2). It is noted that the readout direction of MSC 4 is inverted, i.e. the read-out starts at $790 \mathrm{~nm}$ and proceeds towards shorter wavelengths (see green arrow in Fig. 2). Hence, radiances at the right edge of MSC 4 would be ideal in order to minimize spatial aliasing. But there, spectral correlation was assumed inferior compared to the range overlapping with AVHRR channel 1. The most dominant feature between AVHRR channels 1 and 2 is the absorption by chlorophyll (red edge), and therefore data over land were optionally filtered from the FOV retrieval.

GOME-2 FOVs of nominal and narrow-mode MSC pixels were retrieved from $m=10^{5}$ combined LR/HR measurements. For the PMD FOV, $m=10^{6}$ measurements were col- lected. The time period required for data collection was different for all three different pixel types due to the different measurement frequency and sample size $m$ : (1) 1 to 21 April 2009 for nominal MSC pixels, (2) 23 February, 24 March, and 22 April 2009 for the narrow-mode MSC pixel, and (3) 1 to 25 April 2009 for the nominal PMD pixel. AVHRR data were resampled on two different grids (Sect. 2.2): $4 \mathrm{~km} \times 4 \mathrm{~km}$ for nominal MSC pixels and $1.5 \mathrm{~km} \times 2.5 \mathrm{~km}$ for narrow-mode and PMD pixels.

\subsubsection{OMI/MODIS}

The second LR instrument investigated was OMI (Ozone Monitoring Instrument, Levelt et al., 2006) aboard the NASA Aura satellite (Schoeberl et al., 2006). Compared to GOME2, OMI features a wider swath of $2600 \mathrm{~km}$ covering the entire globe within a day without data gaps. OMI is an imaging spectrometer operated as a push-broom scanner. The UV2/VIS channel of OMI divides the entire swath into 60 individual ground pixels of varying width. The nominal pixel size is $24 \mathrm{~km} \times 13 \mathrm{~km}$ in the nadir direction and increases towards the swath edges.

OMI data recorded after 25 June 2007 are potentially affected by the row anomaly (KNMI, 2015). This instrument anomaly affects level $1 \mathrm{~B}$ radiances depending on viewing angle/pixel number and changes over time. Of particular interest for this study, pixels 53 and 54 (zero-based) are affected since 25 June 2007 and pixels 37 through 42 are affected since 11 May 2008. The anomaly comprises a reduction or increase of the received radiance depending on latitude, with second-order effects. In this study, however, pixels possibly influenced by the row anomaly were included in the FOV retrievals nevertheless.

The OMPIXCOR data set obtained from NASA provides two sets of pixel edges for OMI: (a) tiled pixel edges, whose application results in a seamless swath image, and (b) overlapping $75 F o V$ pixel edges (Kurosu and Celarier, 2010). The across-track widths of both FOV models correspond to the full width at half maximum (FWHM) of the actual FOV. For the $75 \mathrm{FoV}$ pixel edges, the edges are scaled in the along-track direction so that $75 \%$ of the theoretical along-track FOV fall within the pixel edges (Fig. 3). The theoretical alongtrack FOV is calculated by convolving a fourth-order superGaussian with a step function whose length corresponds to the movement of OMI during one pixel integration with respect to the surface (Kurosu and Celarier, 2010). At nadir, both edge definitions produce similar results whereas FoV75 edges are approximately twice as large as tiled edges in along-track direction at the swath edge. It is noted by Dobber et al. (2006) that the polarization scrambler device of OMI imposes non-uniform structures on the spatial response function, which lead to slightly polarization-dependent FOVs.

OMI radiances were compared to radiances recorded by the Moderate Resolution Imaging Spectroradiometer (MODIS) aboard the Aqua satellite (Salomonson et al., 


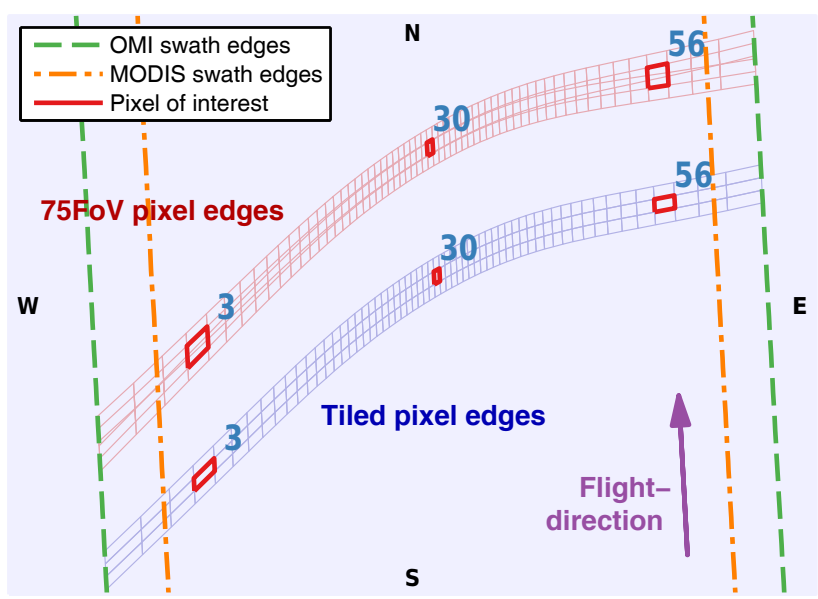

Figure 3. OMI and MODIS swath collocation since May 2008. Furthermore, two OMPIXCOR tiled pixel edges and $75 \mathrm{FoV}$ pixel edges are depicted. Note that the $y$ axis is enhanced by a factor of 4 for the sake of clarity. Pixels highlighted in red are studied in more detail. Pixel numbers are zero-based.

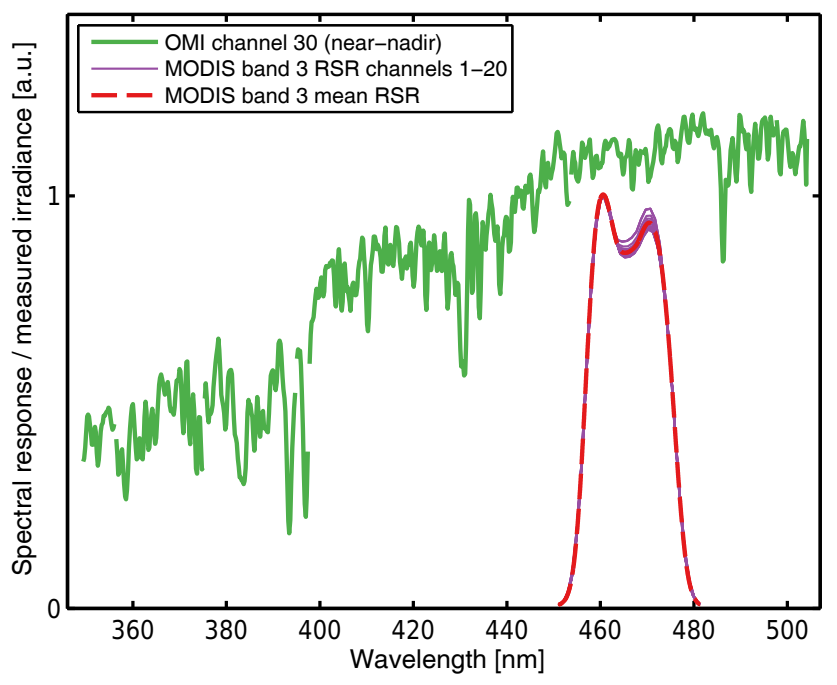

Figure 4. Solar irradiance spectrum measured by the visible channel of OMI on 1 April 2007 and the relative spectral response (RSR) of MODIS Aqua band 3 channels 1 through 20 (Barnes et al., 1998; Xiong et al., 2006, 2008).

1989). MODIS features a swath of $2330 \mathrm{~km}$ width, which is smaller than the OMI swath (Fig. 3). Therefore, it was not possible to evaluate the FOV of all OMI pixels. The outermost OMI pixels included in this study were pixel 3 and 56 at the western and eastern edge of MODIS, respectively. MODIS provides nadir resolutions of $0.25,0.5$, and $1 \mathrm{~km}$ depending on the channel. In this study, MODIS Band 3 (459$479 \mathrm{~nm}$ ) was applied because it provides a favourable resolution of $500 \mathrm{~m}$ in the sensitivity range of OMI as illustrated in Fig. 4.

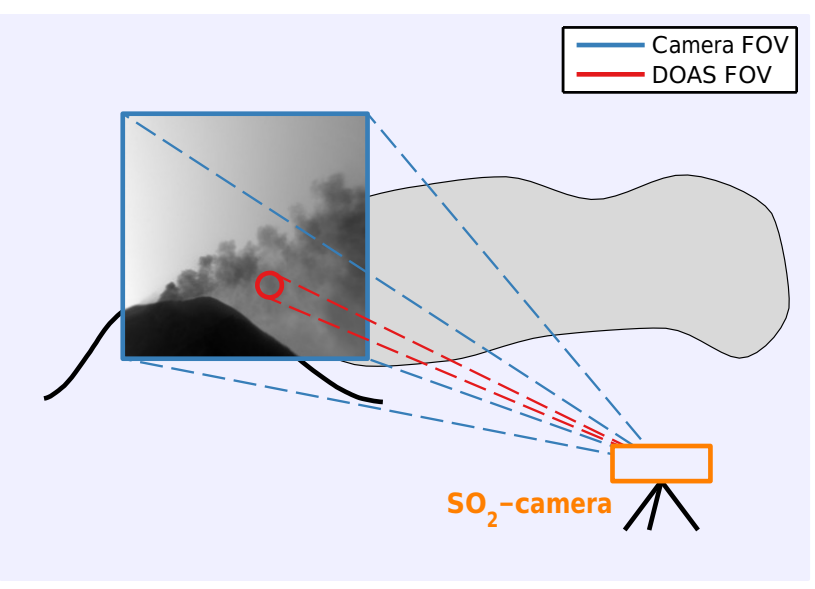

Figure 5. Schematic observation geometry of an $\mathrm{SO}_{2}$ camera with included DOAS device for ground-based observations of volcanic plumes.

Unlike the GOME-2/AVHHR instrument combination, OMI and MODIS are not observing simultaneously. However, Aqua and Aura are both part of the A-train constellation consisting of several spacecraft. Since May 2008, the delay between both observations is approximately $8 \mathrm{~min}$ (Schoeberl, 2002; NASA, 2014). This delay reduces the correlation between OMI and MODIS measurements because cloud scenes and illumination conditions change between overpasses. In order to increase the correlation, combined LR/HR observations with an increased probability of significant cloud movement were filtered by using wind speed interpolated from the global meteorological circulation model maintained by the European Centre for Medium-Range Weather Forecasts (ECMWF). Measurements of scenes in which the interpolated maximum wind speed between 1 and $3 \mathrm{~km}$ altitude exceeds a certain wind-speed threshold $t_{\mathrm{w}}$ were discarded in order to decrease the effect of cloud movement between overpasses. For the retrieval of OMI FOVs, $10^{5} \mathrm{OMI}$ (LR) and coincident MODIS (HR) measurements were collected between -60 and $60^{\circ}$ latitude and between 1 and 9 October 2008 without taking the scene characteristics into account. Combined LR/HR measurements were subsequently filtered using the wind-speed filter. The application of the filter reduced the number of independent observations by $\approx 25 \%$ to $m \approx 7.5 \times 10^{4}$. MODIS data were resampled on a $2 \mathrm{~km} \times 2 \mathrm{~km}$ grid (Sect. 2.2).

\subsubsection{MAX-DOAS/SO 2 -camera}

As third example, IFR was used to characterize the FOV of a ground-based scattered radiation DOAS instrument, which was integrated in an $\mathrm{SO}_{2}$ camera for calibration purposes (e.g. Kern et al., 2010; Lübcke et al., 2013). Figure 5 shows a schematic of the $\mathrm{SO}_{2}$-camera setup for the investigation of volcanic plumes. 
An $\mathrm{SO}_{2}$ camera is an imaging instrument that uses two band-pass interference filters (with a FWHM of approximately $10 \mathrm{~nm}$ ) to measure the optical density of $\mathrm{SO}_{2}$. Images recorded with filter $\mathrm{A}\left(I_{\mathrm{A}}\right)$ measure the optical density of $\mathrm{SO}_{2}$, whereas images recorded with filter $\mathrm{B}\left(I_{\mathrm{B}}\right)$ are used to correct for aerosol influences (Mori and Burton, 2006; Bluth et al., 2007). Furthermore, two respective reference images $I_{\mathrm{A}, 0}$ and $I_{\mathrm{B}, 0}$ with negligible $\mathrm{SO}_{2}$ absorption are required. Then, the apparent absorbance

$\tau=-\ln \frac{I_{\mathrm{A}}}{I_{\mathrm{A}, 0}}+\ln \frac{I_{\mathrm{B}}}{I_{\mathrm{B}, 0}}$

may be calculated (Kern et al., 2010). In the case of negligible ash or aerosol concentrations in the plume, the second term in Eq. 1) vanishes and the first term remains providing the optical density $\tau$ of $\mathrm{SO}_{2}$. In order to calculate $\mathrm{SO}_{2}$ emission rates, the instrument has to be calibrated, i.e. $\tau$ has to be converted to $\mathrm{SO}_{2}$ column densities. This calibration is routinely done with the help of a DOAS spectrometer (e.g. Kern et al., 2010, 2015a; Lübcke et al., 2013; Smekens et al., 2015), in particular for instruments that are permanently installed to monitor volcanoes (Kern et al., 2015b).

The $\mathrm{SO}_{2}$ camera consisted of a CCD detector, a fusedsilica lens and two band-pass interference filters; its properties are summarized in (Kern et al., 2015a) under the name HD-Custom. The complete FOV corresponding to 1024 detector pixels is $23.5^{\circ}$ resulting in $0.023^{\circ}$ per pixel. An OceanOptics USB2000 spectrometer with a narrow FOV ( $400 \mu \mathrm{m}$ fibre diameter, $50 \mathrm{~mm}$ focal length, $0.46^{\circ}$ opening angle) is co-located in the instrument's housing. The two filters were alternatively placed in the light path with a rotating wheel. Images were sequentially acquired with both filters. Filter A measured in a region around $315 \mathrm{~nm}$, where sufficient solar radiation is available and $\mathrm{SO}_{2}$ still has strong absorption features. Filter $\mathrm{B}$ measured around $330 \mathrm{~nm}$, a region where the $\mathrm{SO}_{2}$ absorption is negligible compared to the region of Filter A.

In this study, measurements are taken from the 12th IAVCEI Field Workshop on Volcanic Gases from Lastarria Volcano in Chile $\left(25^{\circ} 10^{\prime} 05^{\prime \prime} \mathrm{S}, 68^{\circ} 30^{\prime} 25^{\prime \prime} \mathrm{W}\right)$ on 21 November 2014. Between 13:39 and 15:30 UTC, a total of $2334 \mathrm{SO}_{2}$ camera images and 2424 LR spectra were recorded, respectively. For data evaluation, the images were reduced to an image size of $512 \times 512$ pixels resulting in $0.046^{\circ}$ per pixel. Since the approximate location and size of the FOV were known, a further subset of $128 \times 128$ pixels was used to determine the exact FOV.

In order to find the FOV of the spectrometer within the camera image, the $\mathrm{SO}_{2}$ optical densities measured by the $\mathrm{SO}_{2}$ camera (HR data) and the DOAS spectrometer (LR data) were compared. Since the $\mathrm{SO}_{2}$ camera and the spectrometer recorded data with a different time resolution, the intensities from the spectrometer were interpolated to match the acquisition times of the $\mathrm{SO}_{2}$-camera images.
The IFR results are compared to the commonly used correlation method introduced by Lübcke et al. (2013) and Smekens et al. (2015). In the implementation of Lübcke et al. (2013), the FOV was found by varying the size and position of an a priori circular FOV disc and calculating the correlation coefficient between the so-called apparent absorbance, i.e. the difference between the optical densities measured with Filter A and Filter $\mathrm{B}$, and the $\mathrm{SO}_{2}$ column density from the DOAS instrument.

\subsection{Resampling of imager data}

The IFR method described below (Sect. 2.3) requires correlated HR/LR measurements. Satellite HR measurements are usually not provided on a discrete, evenly spaced Euclidean grid. Therefore, HR data correlated to each LR measurement need to be resampled to the same regular grid. HR data are required to cover the entire surface surrounding an a priori FOV sampling region. The region may be as large as the entire solid angle of the HR measurement provided it includes the actual FOV of the LR measurement. It is evident that a smaller time difference between HR and LR acquisition time increases their correlation and, hence, increases the SNR of the retrieval. For GOME-2/AVHRR and the $\mathrm{SO}_{2}$ camera both measurements are nearly coincident providing a high correlation. OMI and MODIS, however, are operated from different satellites with different overpass times. Hence, special measures must be undertaken in order to exclude observations with large changes of the radiation field between the overpasses.

Satellite data are typically georeferenced in topocentric logitude/latitude coordinates. These data need to be transformed into an $x / y$ grid relative to the pixel centre with $N_{x}$ and $N_{y}$ grid cells in the $x$ and $y$ direction, respectively. For the sake of simplicity, HR measurements are assumed perfectly geolocated and point-like. In reality, however, HR measurements usually have a FOV size similar to their resolution. The HR resampling involves three steps:

1. Transformation of latitude/longitude/radius coordinates to earth-centred $x / y / z$ coordinates applying the WGS84 ellipsoid ( $x$ axis towards $90^{\circ} \mathrm{E}, y$ axis towards the north pole, $z$ axis towards zero meridian);

2. Rotation of HR data into the $(0,0, z)$-centred $x / y$ plane using the corresponding LR coordinates similar to Siddans (2016):

a. $(x, y, z)$ LR pixel centre (point $\mathrm{F}$ in Fig. 6), around $y$ axis to $\left(0, y^{\prime}, z^{\prime}\right)$,

b. $\left(0, y^{\prime}, z^{\prime}\right)$ around $x$ axis to $\left(0,0, z^{\prime \prime}\right)$,

c. rotation around $z$ axis so that the $y$ offset of both midpoints of the along-track pixel edges $\left(M_{1}\right.$ and $M_{2}$ in Fig. 6b) are equal.

3. Averaging of HR measurements within each grid cell. 
Hence, one HR radiance image is obtained for each LR measurement. The rotations defined in step 2 apply for any pixels of quadrangular shape. Figure 6 shows an example of raw and resampled AVHRR data where the pixel centre and edges of GOME-2 were used as input for the projection. It needs to be noted that the choice of the HR grid is somewhat arbitrary - also irregular grid sizes are possible - but the resolution is constrained by original HR resolution and storage capacity. In this study, quadratic grids are mostly chosen for the sake of simplicity.

AVHRR data were resampled for the FOV retrieval of a variety of GOME-2 pixels. In principle, AVHRR delivers $1.1 \mathrm{~km}$ resolution at nadir, but the across-track resolution becomes poorer at the swath edges. Furthermore, the AVHRR revealed a very weak but systematic, almost alternating radiance offset depending on across-track scan position. This systematic bias perturbed the results because the same LR sub-pixel area was mapped always on the same AVHRR row. The errors became particularly apparent for retrievals at the swath edges. Therefore, a relatively coarse quadratic $4 \mathrm{~km}$ resolution was applied to the retrieval of the FOV of the GOME-2 science channels. Alternatively, the across-track resolution was increased to $1.5 \mathrm{~km}$ for the FOV retrieval of narrow mode and PMD pixels, which were evaluated only in the nadir direction where the distortion in the AVHRR data was found to be negligible.

MODIS data were resampled at a resolution of $2 \mathrm{~km}$ for the retrieval of all OMI FOV within the MODIS swath. In contrast, there was no need to resample $\mathrm{SO}_{2}$-camera images due to its constant alignment to the MAX-DOAS instrument by design. The resolution of the $1024 \times 1024$ full-format images was, however, reduced by a factor of 2 in both spatial dimensions in order to save computing resources.

\subsection{In-operation FOV retrieval (IFR)}

This section formulates the linear equation system (LES) used to invert a FOV pattern from a set of $m$ correlated HR/LR measurements. The $i$ th HR/LR measurement tuple consists of a LR radiance $l_{i}$, which is usually averaged over a selected wavelength range, and the corresponding HR image $h_{i j}$ with $j=1, \ldots, n$, where $n$ is the number of pixels. It is required that the HR image contains the entire LR FOV. If we assume an idealized linear response for both instruments, then $l_{i}$ can be expressed as a linear combination of $h_{i j}$ :

$l_{i}=c_{0}+\sum_{j=1}^{n} h_{i j} c_{j}$

with constant offset $c_{0}$, which adds a further degree of freedom compensating potential input biases due to instrumental deficiencies and imperfect radiance calibration, and discrete FOV coefficients $c_{j}$, which correspond to the fraction of radiation received from each particular solid angle or area within the HR image. Second-order effects necessary to model ef- fects like those caused by the row anomaly of OMI are neglected. Then, all $c_{j}$ with $j=0, \ldots, n$ can be inferred from $m$ measurements by solving

$$
\left(\begin{array}{l}
l_{1} \\
\vdots \\
l_{m}
\end{array}\right)=\left(\begin{array}{llll}
1 & h_{11} & \cdots & h_{1 n} \\
\vdots & \vdots & & \vdots \\
1 & h_{m 1} & \cdots & h_{m n}
\end{array}\right)\left(\begin{array}{l}
c_{0} \\
c_{1} \\
\vdots \\
c_{n}
\end{array}\right)
$$

or, in matrix notation,

$l=\mathbf{H} c$,

where $\boldsymbol{l}$ contains the LR radiances, $\mathbf{H}$ is the $m \times(n+1)$ matrix containing the gridded HR radiances, and $c$ contains the $n+1$ discrete FOV coefficients.

The inversion of Eq. (4), however, is only successful if all input quantities were not significantly affected by measurement errors and $\operatorname{rank} \mathbf{H}=n+1$. In reality, however, all input data contain errors - statistical, systematic, and numeric and different approaches exist to increase the stability of the solution. In this paper, two particular approaches are applied:

1. If the number of linearly independent measurements is $m>n+1$, Eq. (4) yields a least-squares solution of

$\min \|\boldsymbol{l}-\mathbf{H} \boldsymbol{c}\|_{2}$

using standard numerical approaches. For example, the software used for this study applies QR factorization plus column pivoting to solve this numerical problem. The solution gained allows an error estimation described in Appendix A.

2. Sometimes, it may not be possible to acquire a sufficiently large set of measurements, and thus the previous approach is not applicable. Then, however, it is still possible to calculate a solution for $c$ under an additional regularization constraint. In this work, the iterative LSMR method (Fong and Saunders, 2011) is applied to find a solution of the regularized least-squares problem using a regularization parameter $\lambda$. LSMR is a follow-up to the LSQR method (Paige and Saunders, 1982). For this application, the parameter $\lambda$ effectively balances signal-to-noise ratio and spatial bandwidth of the solution. The LSMR solution converges towards the least-squares solution for small $\lambda$ and sufficiently large $m$. The optimal choice of $\lambda$ depends on the application, data quality, and quantity $m$.

The retrieved discrete FOV coefficients $c_{j}$ were finally normalized to unit area of the $x / y$ grid. The resulting FOV fractions

$c_{j}^{*}=\frac{c_{j}}{\Delta x \Delta y \sum_{j} c_{j}}$

correspond to the sensitivity contribution from grid cell $j$ per $\mathrm{km}^{2}$ or per pixel for satellite and $\mathrm{SO}_{2}$-camera application, respectively. 
(a)

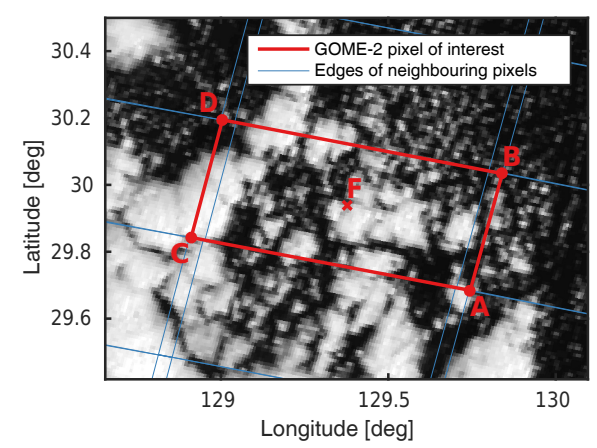

(b)

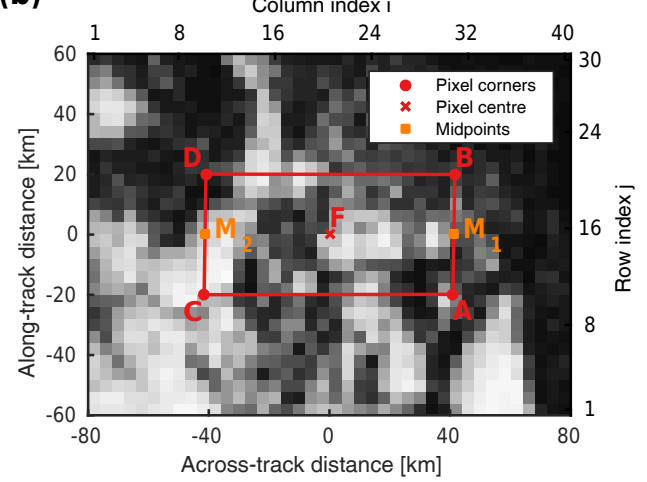

Figure 6. Geospatial alignment of AVHRR measurements and GOME-2 spatial sampling over an example area south of Japan on 2 April 2009: (a) AVHRR raw data in topocentric coordinates, Plate Carrée projection; (b) data projected and averaged on a $4 \mathrm{~km} \times 4 \mathrm{~km}$ grid. The grey levels are extracted from AVHRR channel 1. GOME-2 pixel 12 in the swath centre is highlighted.

\subsection{Parametrized FOV}

For OMI, the discretized FOV results were compared to a parametrized super-Gaussian FOV. Kurosu and Celarier (2010) describe the along-track FOV as the convolution of a flat-topped fourth-order Gaussian IFOV with a boxcar, which was adapted by Kuhlmann et al. (2014). The width of the boxcar is $13 \mathrm{~km}$ corresponding to the travelled distance of the line of sight within one exposure of $2 \mathrm{~s}$. In this study, for the sake of simplicity, the FOV was approximated using a generalized Gaussian model with variable exponent - sometimes referred to as a super-Gaussian model - instead of applying the convolution explicitly. This approximation yields a slightly different FOV shape in the along-track direction. This drawback is outweighed by the advantage of having a single FOV model applicable to all OMI pixels instead of an explicit FOV model depending on viewing angle.

A generalized Gaussian function models the FOV in one dimension

$F_{1-\mathrm{D}}: z_{a}(x)=\gamma \exp \left[-\left|\frac{x-a_{3}}{a_{2}}\right|^{a_{1}}\right]$

with shape parameter $a_{1}$, width $a_{2}$, offset $a_{3}$, and amplitude $\gamma$. It is noted that $F_{1-\mathrm{D}}$ is the Gaussian bell curve for $a_{1}=2$. Equation (7) is enhanced by another dimension and three additional parameters yielding the final two-dimensional FOV expression

$$
\begin{aligned}
F_{2-\mathrm{D}}: z(x, y) & =\gamma z_{a}(x) z_{b}(y) \\
& =\gamma \exp \left[-\left|\frac{x-a_{3}}{a_{2}}\right|^{a_{1}}-\left|\frac{y-b_{3}}{b_{2}}\right|^{b_{1}}\right],
\end{aligned}
$$

where parameter-sets $a_{i}$ and $b_{i}$ describe the FOV shape and position in the $x$ and $y$ direction, respectively. Equation (8) yields seven parameters, which are derived from IFR results $c_{j}\left(j=1 \ldots n, c_{0}\right.$ is discarded $)$ using standard least-squares fitting methods.
It is noted that there are several possibilities to formulate a 2-D superposition of two super-Gaussians. Equation (8) models a rectangular FOV, but it is also possible to simultaneously model skewness and tilt using linear coordinate transformations. Also elliptical FOVs can be realized.

The retrieved widths $a_{2}$ and $b_{2}$ correspond to the $e$-folding lengths of Eq. (7). These parameters are not very intuitive and therefore difficult to compare to the dimensions provided by the OMPIXCOR data (Kurosu and Celarier, 2010). The across-track pixel widths of OMPIXCOR are defined as the FWHM of the FOV. The across-track FWHM $w_{x}$ can be computed using Eq. (7) via

$w_{x}=2 a_{2}(\ln 2)^{1 / a_{1}}$,

where $a_{1}$ and $a_{2}$ are part of the fit output. Likewise, the alongtrack pixel dimension depends on the shape $b_{1}$ and width $b_{2}$, respectively. The $75 \%$ width $w_{y}$ in the $y$ direction fulfilling

$$
\int_{-w_{y} / 2}^{w_{y} / 2} \exp \left[-\left|\frac{y}{b_{2}}\right|^{b_{1}}\right] \mathrm{d} y=\frac{3}{4} \int_{-\infty}^{\infty} z_{b}(y) \mathrm{d} y
$$

corresponds to the definition of the OMPIXCOR $75 \mathrm{FoV}$ along-track width. $w_{y}$ contains three quarters of the received radiance in the along-track direction. Equation (10) was solved numerically. 


\section{Results}

\subsection{GOME-2}

\subsubsection{Main science channel pixels}

This section presents FOV results for GOME-2 pixels 12 (nadir), 0 and 31 (forward scan and backward scan at eastern swath edge), and 23 (western swath edge) as depicted in Fig. 1. The results for all 32 MSC pixels are compiled in the Supplement.

As a first example, the FOV of GOME-2 MSC pixel 12 was characterized for two different convolution kernels. The results are depicted in Fig. 7. A clear rectangular FOV with expected dimension results from evaluating AVHRR channel 1 images and GOME-2 radiances applying a Gaussian convolution kernel centred at $630 \mathrm{~nm}$ (Fig. 7a). The spatial sensitivity inside the FOV is almost constant. The FOV edges, however, have an offset $>15 \mathrm{~km}$, which is reasonably accounted for by the applied spatial aliasing correction (dashed lines). GOME-2 channel 4 data at larger wavelengths are less affected by spatial aliasing due to the shorter readout delay. Hence, switching to $780 \mathrm{~nm}$ reduced the offset between the provided and the interpolated edges (Fig. 7b). Noise, however, increases significantly due to a larger spectral offset between LR and HR data compared to Fig. 7a, even though filtering input data over land could reduce the influence of the spectral offset in Fig. 7b to some extent. The influence of the LR spectral convolution kernel and the number of measurements $m$ is further investigated in Appendix A. For the sake of smaller errors, further results for GOME-2 are obtained applying the $630 \mathrm{~nm}$ Gaussian convolution kernel for GOME-2 data and AVHRR channel 1.

The FOV of the first forward-scan pixel is shown in Fig. 8a. The retrieved FOV shape agrees with the provided pixel edges if spatial aliasing was corrected for. Otherwise, a spatial offset of $\approx 15 \mathrm{~km}$ was observed. The background noise in Fig. 8a is significantly larger than in Fig. 7a even though the number of HR/LR measurements $m$ and the number of FOV nodes $k$ were identical. This is due to a decreased HR/LR correlation, whose potential causes are discussed in Sect. 4.1.

The last forward-scan pixel in Fig. 8b reveals another interesting behaviour: The scan mirror turns within the integration time period of this pixel resulting in a comparatively inhomogeneous sensitivity within the FOV. Furthermore, due to the turning mirror, the spatial aliasing correction only needed to be applied to the eastern pixel edge resulting in a slightly smaller pixel. The retrieved FOV of the last backward-scan pixel 31 is shown in Fig. 9. It reveals an inhomogeneous sensitivity within the FOV again due to a turning scan mirror during integration. The spatial aliasing correction was only applied to the western pixel edge.

\subsubsection{Retrieval error over entire swath}

After the investigation of the FOV of selected GOME-2 pixels, the scan-angle dependence of the reduced residual $\chi^{2}$ of each retrieved FOV (see Eq. A1) is examined. Figure 10 summarizes the $\chi^{2}$ values for all 32 individual MSC pixels in the swath. The plot shows that $\chi^{2}$ is slightly increased for pixels at the swath edges, where the scanning direction changes during integration, i.e. pixels 23 and 31 detailed in Figs. $8 \mathrm{~b}$ and 9 , respectively. More strikingly, Fig. 10 shows that IFR results for pixels 5, 6, and 29 are of much lower quality compared to the other pixels. The respective $\chi^{2}$ peaks in the forward and backward direction are consistently located between -30 and $-25^{\circ}$.

In order to further investigate increased FOV noise levels around $-30^{\circ}$, Fig. 11 shows IFR result for the backwardscan pixel 29. The retrieved FOV is significantly distorted in an along-track band between $x=-40$ and $120 \mathrm{~km}$. This finding may be attributed to instrumental degradation, which is further discussed in Sect. 4.1.

\subsubsection{Nadir narrow-mode MSC and PMD pixels}

Both MSC narrow-mode and PMD FOVs have a nominal size of $10 \mathrm{~km} \times 40 \mathrm{~km}$ in the nadir direction. The observation modes, however, feature different optical paths, different detectors, and different scan mirror speeds. Also the FOV quadrangulars have different shapes - the narrow-mode pixel is more skewed than the PMD pixel - because the integration periods, during which the spacecraft moves, are different for both pixel types by a factor of 8 . For the retrieval of the MSC narrow-mode and PMD FOVs, HR data were resampled to a finer grid of $1.5 \mathrm{~km} \times 2.5 \mathrm{~km}$ resolution to account for the 8 times higher across-track resolution. It is noted that the PMD channel 12 was applied here as it features the best spectral overlap of all GOME-2 PMD channels with AVHRR channel 1 (see Fig. 2).

Figure 12a shows the average nadir FOV of the GOME2 MSC in narrow-mode configuration. At this resolution, the FOV quadrangular is clearly skewed. The PMD FOV in Fig. 12b is much less skewed due to the shorter integration time and, hence, less influence of the satellite motion on the FOV shape.

Furthermore, the distribution of the sensitivity within the FOV was studied using across-track and along-track integrals of the FOV fractions as shown by the magenta lines in Fig. 12a and b. The narrow-mode MSC FOV is characterized by an almost constant boxcar in the along-track direction, whereas the PMD FOV fraction drops by $\approx 5 \%$ for $>10 \mathrm{~km}$. In the across-track direction, both narrow-mode and PMD FOVs reveal smoother edges than in the along-track direction, especially at the eastern pixel edge of the PMD pixel. Furthermore, the PMD pixel appears to be significantly narrower than the provided pixel edges. 
(a)

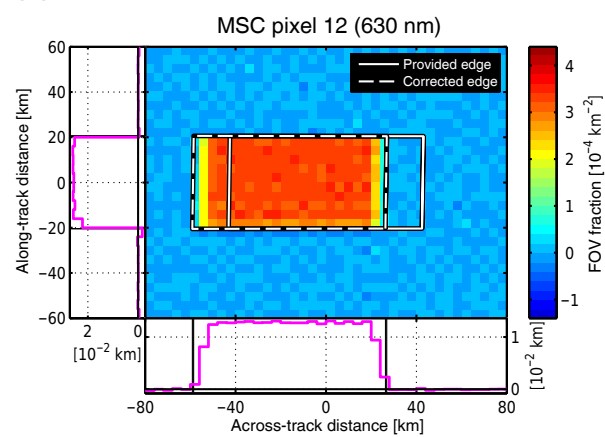

(b)

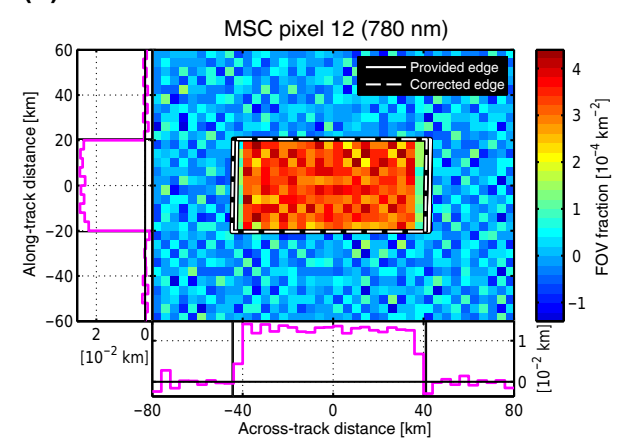

Figure 7. IFR results of GOME-2 MSC pixel 12 (nadir forward scan) at regular swath width inferred for two different LR convolution kernels: (a) Gaussian at $630 \mathrm{~nm}$ and (b) Gaussian at $780 \mathrm{~nm}$. The input data for (b) only include measurements over ocean. The solid line denotes the pixel edges provided with the GOME-2 product, whereas the dashed line denotes the interpolated pixel edges taking spatial aliasing into account. AVHRR channel 1 is applied as HR input for both images. The magenta lines in the panels below and left of the 2-D FOV result denote across- and along-track integrals of the 2-D results, respectively.

(a)

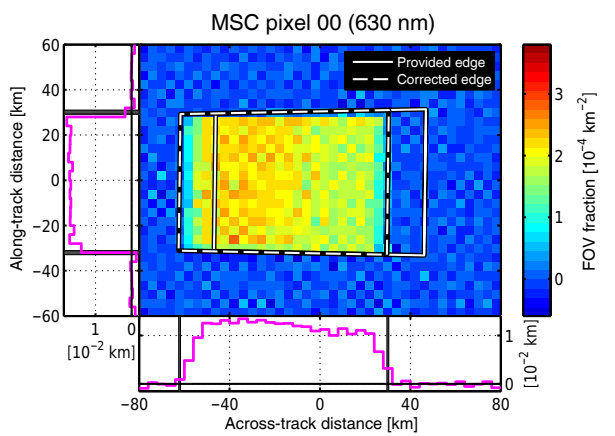

(b)

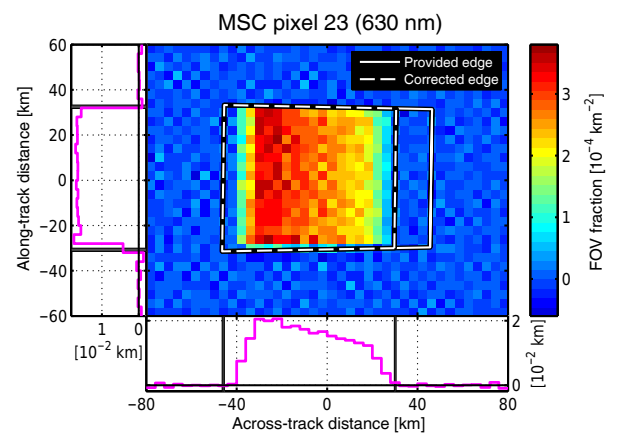

Figure 8. As Fig. 7a but for GOME-2 MSC swath edge pixels: (a) pixel 0 (first forward scan), and (b) pixel 23 (last forward scan). Note that the spatial aliasing correction in (b) is only applied for the eastern across-track edge as the mirror turns during integration.

\subsection{OMI}

The design of the OMI satellite instrument is fundamentally different from GOME-2 as described in Sect. 2.1.2. Furthermore, the HR measurements by MODIS are taken 8 min after the OMI measurements deteriorating the correlation between both measurements. Therefore, the initial set of $10^{5} \mathrm{com}-$ bined measurements was filtered using a wind-speed threshold $t_{\mathrm{w}}=15 \mathrm{~m} \mathrm{~s}^{-1}$ which proved to increase SNR of the FOV retrieval significantly. The choice of $t_{\mathrm{w}}$ was determined in preceding tests and presents a trade-off between discarding too many measurements, which would decrease SNR, and not filtering enough measurement necessary to reduce the smearing effect of scene changes due to cloud movement. The results can be considered robust as they only weakly depend on $t_{\mathrm{w}}$.

Figure 13a shows the FOV results using the numerically exact LES solver. The SNR is much poorer compared to the result obtained from a similar sample size of GOME2/AVHRR data (see Fig. 7). Therefore, the iterative LSMR method was applied as a fallback method because it com- putes approximate results while damping high spatial frequencies, and, hence, yields reduced retrieval noise. LSMR requires an additional parameter $\lambda$, which was empirically determined (see Sect. 3.2.2). Figure 13b through d show the retrieved FOV of pixel 30 in the nadir direction depending on $\lambda$. The noise decreased with increasing $\lambda$. The result for $\lambda=1 \times 10^{-3}$ (Fig. 13d) is comparatively smooth and the FOV distribution is almost 2-D Gaussian. The dependence on $\lambda$ is investigated in more detail in Sect. 3.2.2.

\subsubsection{Parametrized FOV}

To compare OMI FOV shapes quantitatively, the retrieved FOV fractions were used as input to fit the FOV parametrization of Eq. (8). An example fit output corresponding to Fig. 13c (OMI pixel 30, $\lambda=3 \times 10^{-4}$ ) is compiled in Fig. 14 . The residual in Fig. 14b indicates that the fit succeeded in finding a reasonable solution without significant contributions not captured by the FOV parametrization.

Figures $14 \mathrm{c}$ and $\mathrm{d}$ show the integrated FOV cross sections in the across- and along-track direction, respectively. The 


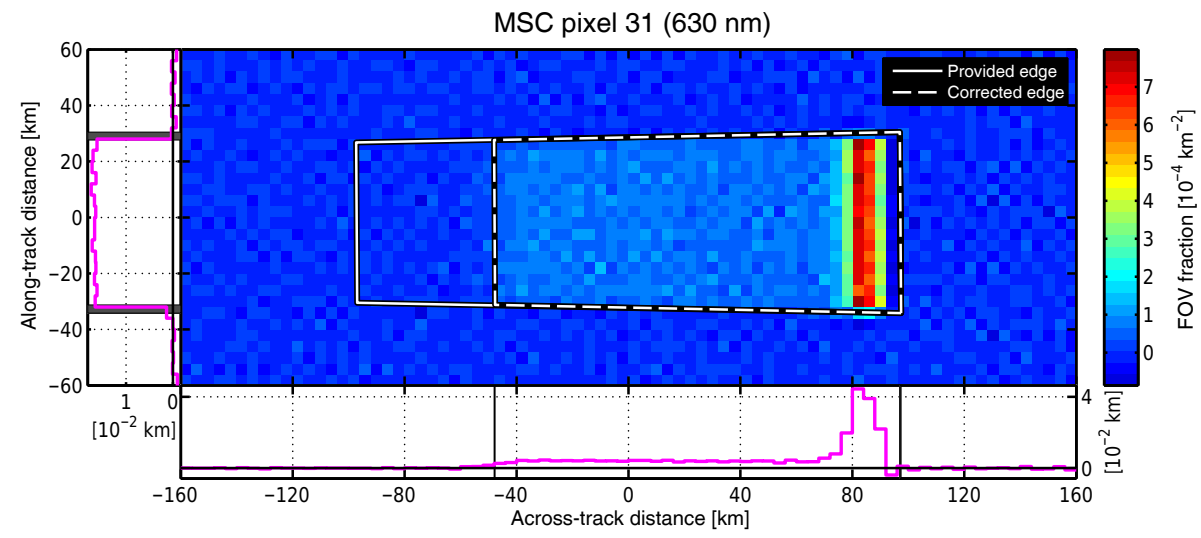

Figure 9. As Fig. 7a but for GOME-2 MSC pixel 31 (last backward scan). Note that the spatial aliasing correction is only applied for the western across-track edge as the mirror turns during integration.

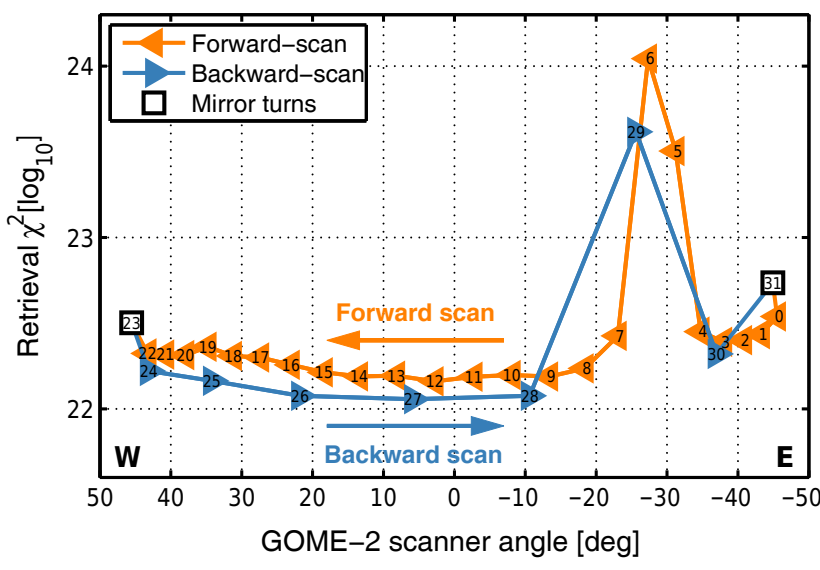

Figure 10. Retrieved $\chi^{2}$ depending on GOME-2 scanner angle and pixel number (see Fig. 1) for the entire swath. The FOV residual increases between -30 and $-25^{\circ}$ potentially indicating telescope pointing instabilities in this range. The respective FOV result images are compiled in the Supplement.

shape of the retrieved FOV is well reproduced by the 2-D fit (red line). However, there is a difference of shape, amplitude, and position between the theoretical and retrieved along-track FOV in Fig. 14d due to the simplifications of the parametrized FOV model.

\subsubsection{Dependence on $\lambda$}

To test whether the fit results presented above are representative, the $\lambda$ dependence of the fitted FOV shape and width were investigated (Fig. 15). It is noted that the widths in the across- and along-track direction $w_{x}$ and $w_{y}$, respectively, were defined differently (Sect. 2.4). In the across-track direction, the FWHM width $w_{x}$ as defined by Eq. (9) is shown. In the along-track direction, however, the $75 \%$ width $w_{y}$ was defined in analogy to the OMPIXCOR $75 \mathrm{FoV}$ pixel edge for better comparability. Equation (10) was used to calculate $w_{y}$ from the fit results.

In the $x$ direction, the shape parameter $a_{1}$ features a plateau around 3.5 for $\lambda \leq 3 \times 10^{-4}$ before decreasing with increasing $\lambda$. The across-track width $w_{x}$ is almost constantly $24 \mathrm{~km}$ for $\lambda \leq 1 \times 10^{-3}$ before increasing significantly towards larger $\lambda$.

In the $y$ direction, the shape $b_{1}$ is again almost constant for $\lambda \leq 3 \times 10^{-4}$ at 2.1. Above $\lambda=3 \times 10^{-4}, b_{1}$ increases slightly peaking at 2.3 at $\lambda=3 \times 10^{-3}$. The alongtrack width $w_{y}$ shows a dependence on $\lambda$ similar to $w_{x}$, remaining almost constant for $\lambda \leq 1 \times 10^{-3}$ and then increasing rapidly.

Smaller $\lambda$ result in decreased SNR, larger $\lambda$ reduce the resolution with smaller shape and larger width parameters. Hence, $\lambda=3 \times 10^{-4}$ is assumed as a reasonable trade-off between noise and spatial resolution (see Fig. 13). It is noted that the observed behaviour of fitted shape and width was almost independent of $t_{\mathrm{w}}$. For $t_{\mathrm{w}}<15 \mathrm{~m} \mathrm{~s}^{-1}$, shape parameters become slightly larger and widths are slightly (less than $1 \mathrm{~km})$ smaller but the noise increases due to reduced statistics. Therefore, parameters $\lambda=3 \times 10^{-4}$ and $t_{\mathrm{w}}=15 \mathrm{~m} \mathrm{~s}^{-1}$ were chosen for the results of the complete OMI swath (pixel 3-56), which are compiled in the Supplement.

\subsubsection{Viewing angle dependence}

In the following, the results for both extreme east and west viewing directions within the MODIS swath are presented. Figure 16a and $\mathrm{b}$ display the FOV of OMI pixels 3 (west) and 56 (east), respectively. Compared to pixel 3, the sensitivity of pixel 56 seems to be more heterogeneous and the background noise is larger. Both results reveal background structures, which are periodic in the along-track direction and probably caused by the multiple use of overlapping HR data corresponding to neighbouring LR pixels. The FOV maximum in Fig. $16 \mathrm{~b}$ is approximately at $x=-30 \mathrm{~km}$ indicating an asymmetry in the across-track direction. Figure 16a and $b$ 


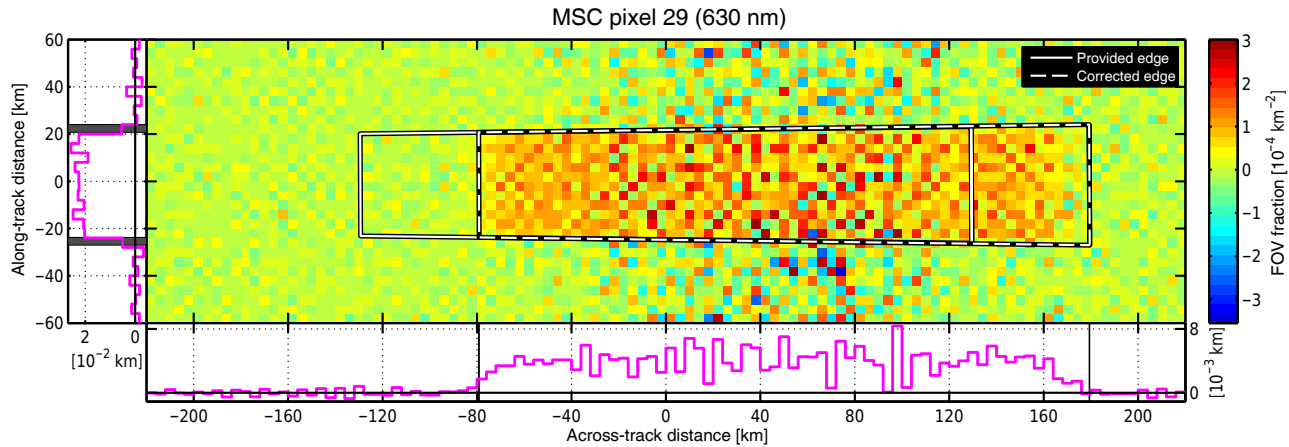

Figure 11. As Fig. 7a but for GOME-2 MSC pixel 29 revealing the strongest FOV distortions in the backward scan.

(a)

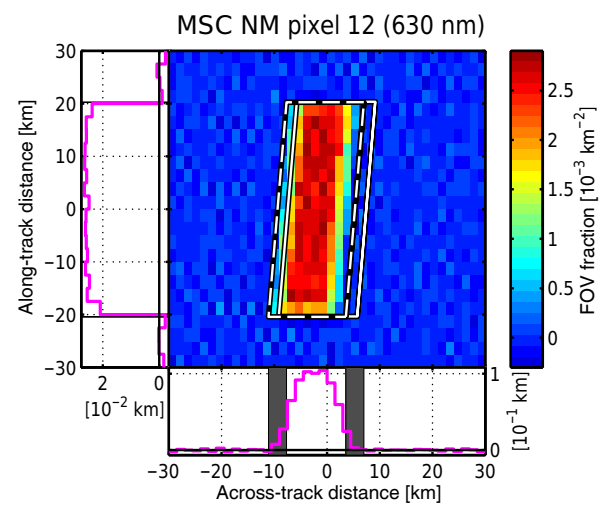

(b)

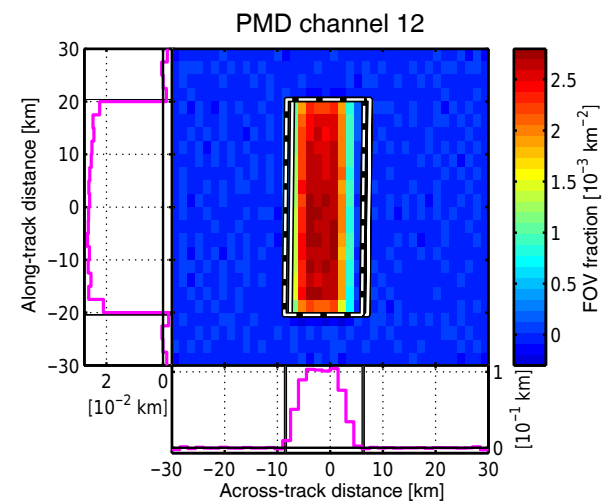

Figure 12. As Fig. 7a but (a) for an average MSC narrow-mode (NM) pixel at nadir (forward scan) and (b) for an average PMD-PP channel 12 pixel at nadir (forward scan). Note the different spatial resolution of $1.5, \mathrm{~km} \times 2.5 \mathrm{~km}$.

furthermore show an increase of the integrated across-track FOVs (magenta line in the bottom panels) towards the borders $(x= \pm 120 \mathrm{~km})$. This behaviour becomes increasingly visible towards the swath edges (see Supplement) and is discussed in Sect. 4.2.

The FOV parametrization Eq. (8) was fitted to the FOV results from pixel 3 through 56 (see Sect. 3.2.1 and Supplement). Figure 17 compiles the results for all three parameter classes: shape, width, and spatial offset with respect to the provided pixel centre in both directions, respectively. The shape parameter $a_{1}$ scatters around a minimum 3.5 in the swath centre and increases slowly to 4 and 4.5 at the western and eastern swath edge, respectively. In the along-track direction, $b_{1}$ averages to 2.2 with comparatively small scatter and negligible viewing angle dependence.

The FOV widths show little scatter and depend on the viewing angle as expected (Fig. 17b). There are, however, differences when compared to the pixel widths provided in OMPIXCOR. In the across-track direction, differences between retrieved FWHM width $w_{x}$ and provided tiled pixel edges are negligible. In the along-track direction, however, there are systematic differences. The retrieved $75 \%$ width is $\approx 1 \mathrm{~km}$ larger than $75 \mathrm{FoV}$ from OMPIXCOR at small view- ing angles, whereas pixels at the swath edge (3-10 and 5056) appear even narrower than provided.

Figure $17 \mathrm{c}$ reveals that the OMI pixel FOVs were systematically shifted in both dimensions. The displacement in the across-track direction $a_{3}$ is negative outside the range between 10 and $30 \mathrm{~km}$, i.e. the actual centre of the FOV was shifted westward (see Fig. 16b). The absolute across-track offset was maximal $(>1 \mathrm{~km})$ at the swath edges. The viewing angle dependence of the spatial offset in the along-track direction $b_{3}$ was clearly more complex, but still systematic and smooth. For the entire swath, $b_{3}$ is in the range between 1 and $1.5 \mathrm{~km}$. At the swath edges, $b_{3}$ was always positive, i.e. a displacement towards the north, which is visible in Fig. 16.

\section{3 $\quad \mathrm{SO}_{2}$ camera}

The applicability of IFR to ground-based measurements is demonstrated using $\mathrm{SO}_{2}$ apparent absorbance and $\mathrm{SO}_{2}$ SCDs measured by an $\mathrm{SO}_{2}$ camera and a DOAS instrument, respectively. The iterative LSMR method was applied $\left(\lambda=2.5 \times 10^{-4}\right)$ because the number of correlated HR/LR measurements was limited. Furthermore, the same data were evaluated applying the correlation method for comparison 
(a) Exact solution

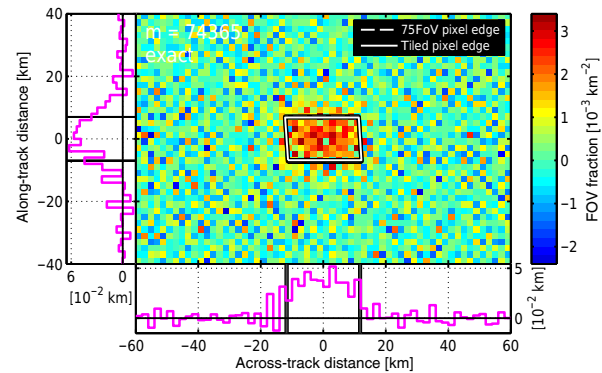

(c) $\operatorname{LSMR}\left(\lambda=3 \times 10^{-4}\right)$

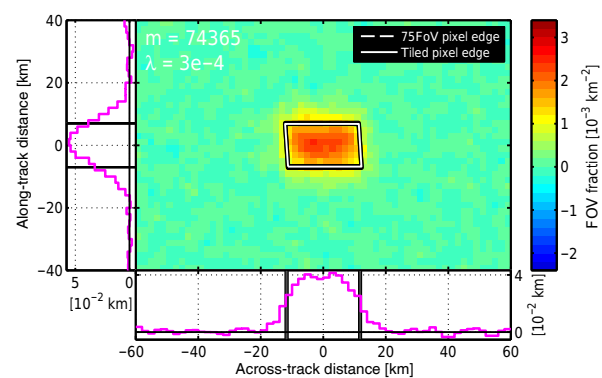

(b) $\operatorname{LSMR}\left(\lambda=2 \times 10^{-4}\right)$

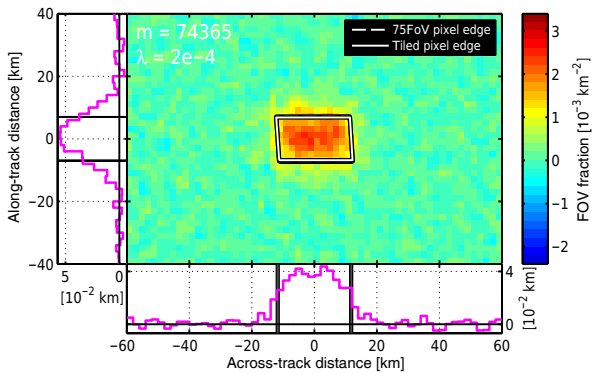

(d) $\operatorname{LSMR}\left(\lambda=1 \times 10^{-3}\right)$

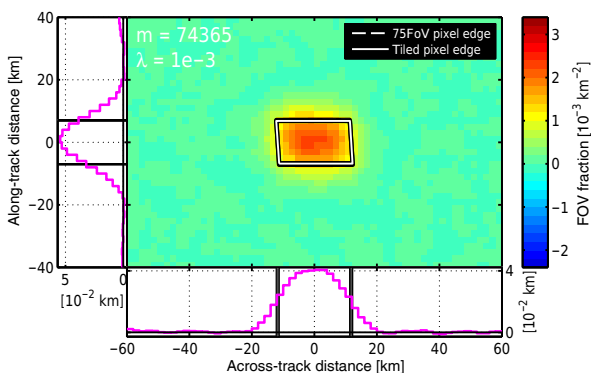

Figure 13. IFR results of OMI VIS channel pixel 30 (nadir) using different settings for the numerical inversion: (a) numerically exact solution, and (b) through (d) approximate LSMR solutions applying regularization parameters $\lambda=2 \times 10^{-4}, 3 \times 10^{-4}$, and $1 \times 10^{-3}$, respectively $\left(t_{\mathrm{W}}=15 \mathrm{~m} \mathrm{~s}^{-1}\right)$. It is noted that, for this observation geometry, the two OMI pixel coordinate products $75 \mathrm{FoV}$ (dashed line) and tiled (solid line) are almost identical.

(a) Fitted 2D Super-Gaussian

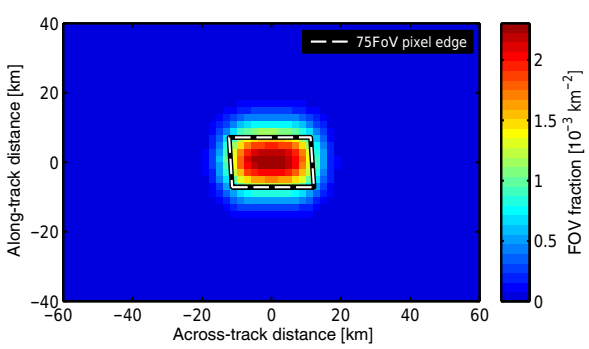

(c) Across-track FOV

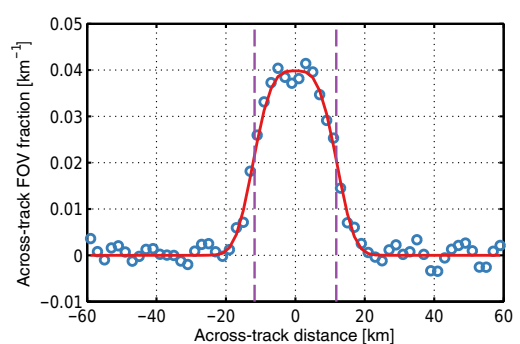

(b) Fit residual

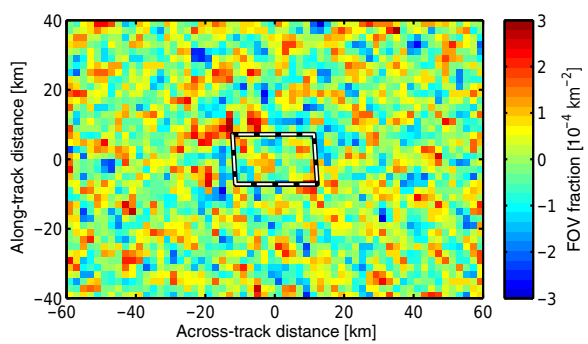

(d) Along-track FOV

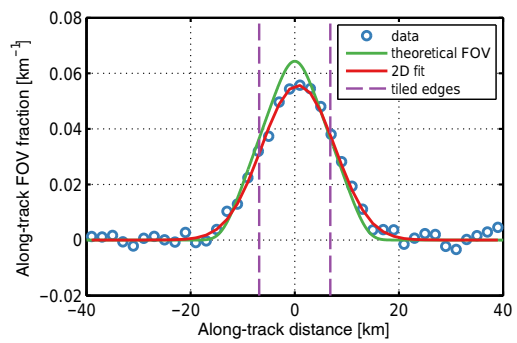

Figure 14. Fit of the nadir FOV results of OMI in Fig. 13c: (a) fit result of the 2-D FOV model in Eq. (8), (b) fit residual, (c) retrieved across-track FOV cross section with 2-D fit result compared to tiled pixel edges, and (d) retrieved along-track FOV cross section with 2-D fit result compared to theoretical FOV shape and tiled pixel edges $\left(\lambda=3 \times 10^{-4}, t_{\mathrm{W}}=15 \mathrm{~m} \mathrm{~s}^{-1}\right)$. Note that (c) and (d) compare integrated results (blue dots correspond to the magenta lines in Fig. 13c), fit results, and theoretical FOV shape in the across- and along-track direction, respectively. 
(a)

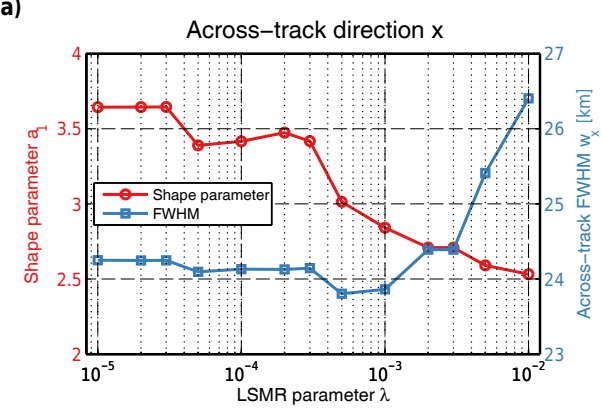

(b)

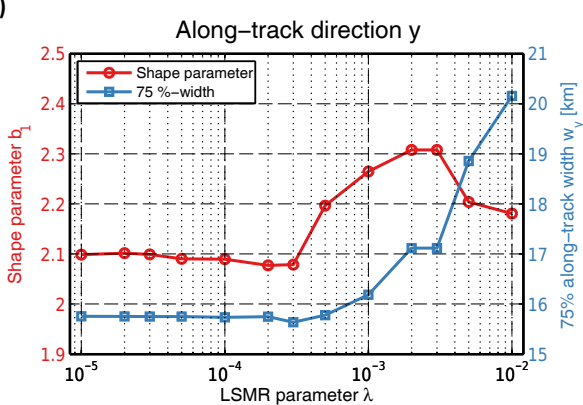

Figure 15. Fitted FOV shape and width for OMI pixel 30 (Fig. 13) depending on LSMR regularization parameter $\lambda$ in the (a) across- and (b) along-track direction, respectively $\left(t_{\mathrm{W}}=15 \mathrm{~m} \mathrm{~s}^{-1}\right)$.

(a)

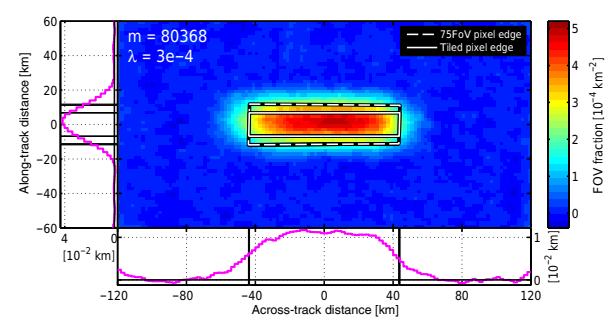

(b)

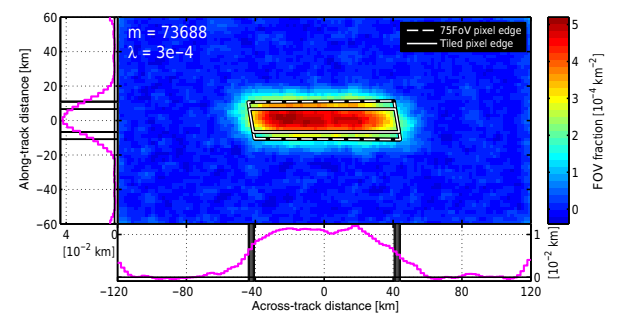

Figure 16. As Fig. 13c but for OMI pixel 3 (western MODIS swath edge) and pixel 56 (eastern swath edge). Note that the retrieval ranges are adjusted to the increased nominal FOV size.

(Sect. 2.1.3). Figure $18 \mathrm{~b}$ shows the results of both methods. The example $\mathrm{SO}_{2}$ apparent absorbance image in Fig. 18a exhibits a linear distortion along the edge of the mountain (row number 240 and below). This distortion was caused by slight changes of the light path when different filters were applied.

For IFR, the size of the FOV was determined by fitting a Gaussian to the horizontal and vertical cross section through the peak of the FOV result. The fit results then yielded a horizontal FWHM of $0.49^{\circ}$ and a vertical FWHM of $0.42^{\circ}$. For comparison, the correlation method obtained an almost perfect HR/LR correlation for a circular FOV with an angle of view diameter in the range between 0.4 and $0.9^{\circ}$ with a maximum at $0.6^{\circ}$. The maximum found by IFR seems to be significantly biased towards the top right. Numerically, however, the bias was small (0.35 and 1.2 pixel in the horizontal and vertical direction, respectively) if the fit results of the two Gaussians above were applied. This behaviour may be due to the minor contributions in the retrieved FOV distribution to the lower left of the black circle.

\section{Discussion}

\subsection{GOME-2}

The FOV results for GOME-2 confirm that most of the actual measurement sensitivity is confined to the pixel edges provided by EUMETSAT - as long as spatial aliasing is cor- rected for. The spatial aliasing correction depends on the wavelength range of interest and, hence, on the retrieval of various properties, e.g. trace gas column, aerosol index, or cloud fraction. It is noted that the spatial aliasing effect for most retrievals is smaller than illustrated in Fig. 7a because the Gaussian $630 \mathrm{~km}$ convolution kernel applied covers the left edge of MSC channel 4, which is read out last.

For the AVHRR/GOME-2 combination, HR and LR measurements are highly correlated because both instruments are mounted on the same spacecraft, and therefore the temporal offset in the data is minimal. Different spectral convolution kernels for the LR measurements were tested in the course of this study and, surprisingly, the highest SNR has not been achieved using the spectral response of AVHRR channel 1 (see Figs. 2 and A1). Instead, the highest SNR has been achieved using a Gaussian-shaped convolution kernel centred at $630 \mathrm{~nm}$ with a FWHM similar to the spectral response of AVHRR channel 1.

The influence of spatial aliasing was minimized by using a spectral convolution kernel at the right edge of GOME2 channel 4 where the detector readout starts. Therefore, a synthetic spectral response peaking at $780 \mathrm{~nm}$ was used to convolve LR measurements. The retrieval noise was found larger than with the above settings, even though measurements over land were filtered in order to reduce interferences with chlorophyll absorption. Appendix A compiles a matrix 


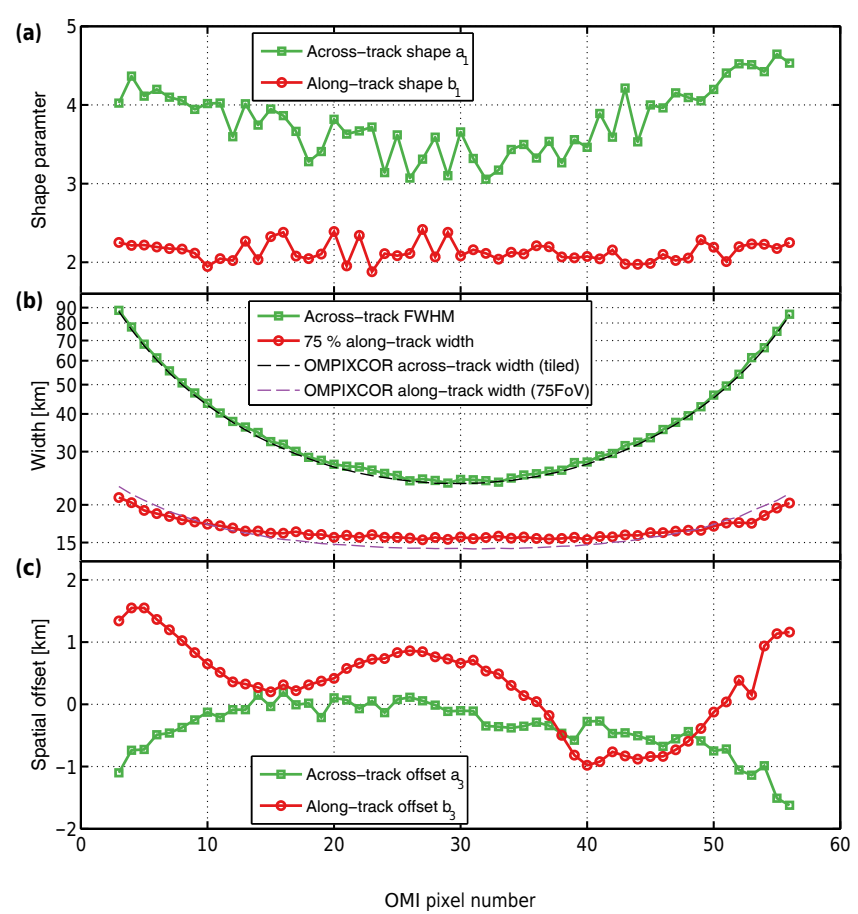

Figure 17. Dependence of the retrieved FOV parametrization on OMI pixel number in the across-track (green squares) and along-track (red circles) direction, respectively $\left(\lambda=3 \times 10^{-4}\right.$, $t_{\mathrm{W}}=15 \mathrm{~m} \mathrm{~s}^{-1}$ ): (a) Shape parameters $a_{1}$ and $b_{1}$, (b) retrieved FOV widths compared to corresponding tiled and $75 \mathrm{FoV}$ pixel widths provided by OMPIXCOR, and (c) spatial offsets $a_{3}$ and $b_{3}$.

analysis between different LR convolution kernels and HR channels and discusses the FOV retrieval error.

Furthermore, the FOV at the swath edge and for special scanning modes has been investigated. At the edge of the standard $1920 \mathrm{~km}$ swath, the FOV distributions of the MSC pixels are less homogeneous than in the nadir direction. In particular, the moving direction of the scan mirror changes during the integration of pixels 23 and 31 creating complex FOV distributions. Depending on the spatial aliasing, pixel 31 (last back-scan pixel) provides the best spatial resolution of all GOME-2 MSC pixels, which is, however, seldom used because most retrievals remove back-scan pixels from further processing by default.

It is observed that the FOV contributions decrease towards the swath edges in pixels 23 and 31. Furthermore, also the FOV for pixel 0 in Fig. 8a shows a significant gradient in the scan direction. It seems that the scan-mirror does not reach the intended turning point, maybe due to accumulated lubricant in the bearing of the stepper motor. Then, the uneven FOV pattern of pixel 0 could be explained by a jitter of the actual mirror position compared to the intended mirror position. The accumulation of lubricant at both turning points of the scanner after a certain period of operation is a known issue of the instrument design of GOME-2. Therefore, a con- tinuous $360^{\circ}$ mirror spinning during the night side of the orbit has been introduced by GOME- 2 operations in 2008 in order to mitigate the lubricant build-up. The spinning improved the mirror positioning statistics of GOME-2 as a whole (see daily reports on EUMETSAT, 2015a), but at the swath edges in particular, the mirror spinning is mitigating the issue not completely.

The residual $\chi^{2}$ is not equal for all pixels. Especially pixels 5, 6, and 29 suffer from an inferior SNR compared to the other pixels (Fig. 10). Furthermore, $\chi^{2}$ increased towards the swath edges. The reasons for the inferior SNR can be manifold. (a) One explanation could be that the mirror does not travel very smoothly in this viewing angle range leading to significant pointing error (jitter) which, in turn, would reduce the correlation for IFR. However, the position difference and current drawn by the stepper motor do not indicate a systematic problem between -30 and $-25^{\circ}$ scanner angle (EUMETSAT, 2015a). (b) Another hypothesis for the observed distortions, sun glint, could be ruled out during preliminary tests where observations over ocean were excluded. (c) There may still be another reason for the increased noise at the swath edges. The resolution of the stepper motor at the swath edges is inferior to that at nadir because the motor speed is variable to maintain a regular pixel size in the acrosstrack direction (Munro et al., 2016). A pixel at the swath edge features less stepper motor steps and, hence, pointing accuracy decreases and positioning jitter increases as observed. (d) Erroneous AVHRR imager data are another possibility. A viewing-angle-dependent radiometric or pointing instability would propagate into the FOV results. For example, preliminary evaluations of the AVHRR data revealed systematic column-by-column variations which may interfere with IFR. The angular velocities of LR and HR instruments are different, which may increase viewing-angle-dependent interferences even further.

As last examples for GOME-2 pixel shapes, the FOV of MSC narrow-mode and PMD pixels were investigated (Fig. 12). Periodic structures, as in Fig. 16 for OMI, are evident in neither the along- nor across-track direction, even though always nine neighbouring pixels within one scan were used. The FOV width in the across-track direction of the PMD and narrow-mode nadir pixels are similar. This observation is surprising because the across-track PMD pixel edges were assumed much steeper. Furthermore, the observed across-track FOV shape is ascribed to the readout of the PMD channel 12. The total PMD readout lasts longer than the readout of a single PMD detector channel $(45.776 \mu \mathrm{s})$ due to the binning within each PMD channel as defined in Lang (2010). Another parameter leading to smoother edges in the across-track direction may be the convolution with an IFOV width of $4 \mathrm{~km}$, which is much less prominent at regular swath widths.

For the PMD channel, the spatial aliasing effect is less prominent compared to the MSC examples due to the different readout timing. It is furthermore observed that the 
(a) $\mathrm{SO}_{2}$ apparent absorbance

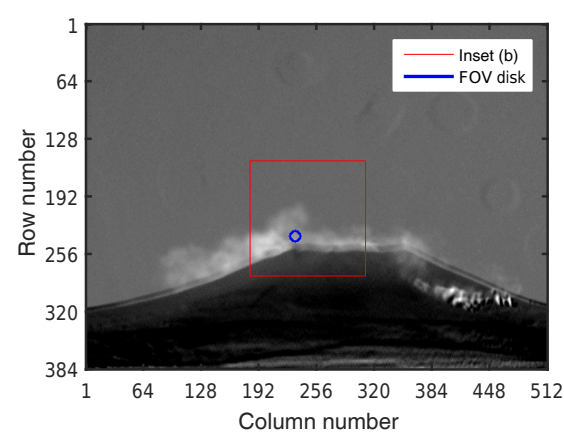

(b) FOV results

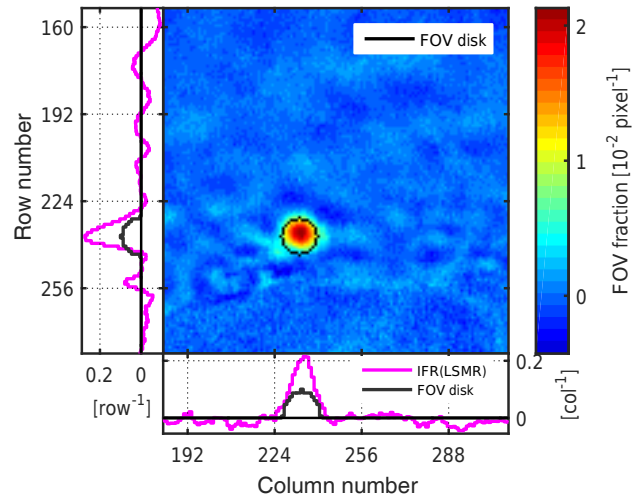

Figure 18. $\mathrm{SO}_{2}$-camera data recorded on 21 November, 2014 at Lastarria Volcano: (a) example apparent absorbance $\tau$ measured at 13:39:30 UTC (white areas correspond to large $\mathrm{SO}_{2}$ column densities) illustrating the HR FOV, (b) comparison of retrieved FOV using the correlation method $\left(0.6^{\circ}\right.$ diameter FOV disc, circle is outer range) and IFR applying the LSMR method $\left(\lambda=2.5 \times 10^{-4}\right)$. The resolution of the $\mathrm{SO}_{2}$-camera image is 512 pixel per $23.5^{\circ}$ corresponding to $0.046^{\circ} /$ pixel.

across-track PMD FOV distribution is significantly narrower than suggested by the pixel edges and that it is shifted in the scanning direction (to the left). However, satellite retrievals relying on an accurate mapping of AVHRR cloudfraction data on GOME-2 PMD pixels like the Polar Multisensor Aerosol Product (PMAp EUMETSAT, 2015b) are potentially affected by the FOV differences found.

The FOV integrated in the along-track direction differs between PMD and narrow-mode pixels. While the narrowmode FOV is as flat-topped as for regular MSC pixels (see Fig. 7a), the PMD FOV features a statistically significant variation of approximately $5 \%$. This variation may be attributed to the different optical paths in the GOME-2 instrument possibly leading to different effective sensitivities across the aperture of the instrument.

It is finally noted for the $10 \mathrm{~km} \times 40 \mathrm{~km}$ FOVs that the pointing instabilities discussed above may have a minor impact on the MSC narrow mode alignment because the mirror moves slower. For the PMDs, however, the resulting distortions can be assumed more significant due to the 8 times higher resolution in the across-track direction.

\subsection{OMI}

The retrieval of FOV for the OMI instrument is more complicated than for GOME-2. The application of a wind-speed filter increases the correlation between HR and LR measurements significantly. Despite the filter, however, the exact results still have higher noise levels, probably due to the orbital delay between the Aqua and Aura satellites. Therefore, an approximating numerical solver is applied, which also acts as a spatial low-pass filter.

The retrieved FOV shape and size are very close to the parameters prescribed in the technical documentation. Kurosu and Celarier (2010) assume a fourth-order Gaussian as an along-track IFOV for OMI, which is then convolved with the platform movement during integration. This convolution was approximated by directly fitting a 2-D super-Gaussian, which was found to describe the retrieved OMI FOV features very well. The differences between convolved and approximated version are minor (see Fig. 13d), and the fitted superGaussian seems to even better represent the retrieved FOV values than the theoretical FOV shape. In principle, the OMPIXCOR pixel edges suggest a skewed 2-D super-Gaussian. However, the IFR results obtained for OMI are not significantly skewed. The proposed 2-D super-Gaussian therefore appears to be a sufficient approximation in this study, which could be implemented into standard gridding routines for OMI.

At the swath edge, the two provided pixel edges (tiled and $75 \mathrm{FoV}$ ) deviate significantly and the $75 \mathrm{FoV}$ pixel edges apparently capture the retrieved FOV much better than the tiled pixel edges. In the swath centre, the retrieved $75 \%$ alongtrack widths are larger than the provided $75 \mathrm{FoV}$ widths, whereas the opposite is the case at the swath edges. It must be noted that the presented results probably overestimate small FOV widths due to the effective smoothing of the LSMR solver in combination with residual cloud movement. It is therefore surprising that the provided $75 \mathrm{FoV}$ width actually seems to overestimate the true along-track width at the swath edges. This overestimation, however, only plays a minor role in the application of OMI data because many studies discard measurements with pixel numbers smaller than 10 and above 50 due to their inferior spatial resolution. It is furthermore noted that the provided FWHM widths in the across-track direction are perfectly reproduced by this study.

Furthermore, there is a systematic spatial offset of the FOV centre depending on the viewing angle. The offset is of the order of $\pm 1 \mathrm{~km}$ in both directions, which is still within the instrument specification. The observed offset is apparently 
due to wavelength-dependent properties of the OMI optics in general and the diamond effect of the polarization scrambler in particular. It is noted that the temporal stability of the OMI geolocation offset is of the same order as investigated by Kroon et al. (2008) during the years 2005-2006.

Towards the swath edges, a typical behaviour of IFR applying LSMR may be observed: The FOV increases towards the domain edges producing small wings of the along-track integrals (plotted in magenta at the bottom of both Fig. 16a and $b$ ). One possible explanation for this behaviour may be atmospheric or instrumental straylight at these viewing angles. Numerical simulations showed that additional random contributions, which are not captured by the applied linear FOV model, can lead to this type of behaviour. Consequently, strong interferences for pixels affected by the row anomaly, which appeared later during the mission, were observed in preceding IFR experiments conducted at various grid sizes and resolutions. These results were not included into this paper due to the ambiguity of the interpretation. However, the FOV results of pixels 37-42 and 53-54 (see Supplement) are not significantly affected by the row anomaly even though the measurements are actually affected according to KNMI (2015). Apparently, the influence of the row anomaly on IFR based on data recorded in October 2008 is negligible. In order to investigate the effect of row anomalies in the future, second-order effects may be added to the linear FOV model applied in this paper.

It is finally noted that the results obtained for OMI in this study are in accordance to the FOV parameters derived by de Graaf et al. (2016), who presented a similar 2-D parametrization of the OMI FOV derived from correlated MODIS measurements. The main difference between this work and de Graaf et al. (2016) is that we retrieved a discretized FOV prior fitting a 2-D distribution, which required orders of magnitude more HR measurements. Hence, FOV structures different to an a priori FOV parametrization could, in principle, be retrieved. Furthermore, the FOV parametrization applied by de Graaf et al. (2016) did not include any spatial shifts and parameters were obtained by looking for maximum correlation while changing the coefficients rather than by applying a least-squares fit. For OMI, however, the agreement between both approaches confirms the validity of using a FOV model with only few parameters, which may not have been the case a priori.

\section{3 $\mathrm{SO}_{2}$ camera}

The FWHM values from the IFR results $\left(0.49\right.$ and $\left.0.42^{\circ}\right)$ are at the lower end of the range obtained by the correlation method $\left(0.4\right.$ to $\left.0.9^{\circ}\right)$ and compare very well to the theoretical FOV of $0.46^{\circ}$. Concerning the FOV shape, the results in Fig. 18b indicate some distortion from the flat disc approximation assumed for the correlation method. The IFR result reveals an almost circular peak blurred to the lower left. The apparent FOV contribution at the bottom left of the circle may be caused by a combination of temporal delay between HR and LR measurements as well as acquisition delay between $I_{\mathrm{A}}$ and $I_{\mathrm{B}}$ since the plume was moving into that direction. Furthermore, distortions at the mountain edge below row number 240 could have an influence. It is therefore difficult to judge whether this contribution to the FOV is real or not. Furthermore, the spatial smoothing caused by the LSMR method adds another uncertainty because $m=2334$ was much smaller for the $\mathrm{SO}_{2}$ camera compared to satellite measurements and therefore it was not possible to retrieve the FOV at such a high spatial resolution. The size and shape of the artefacts are similar to the background noise structures whose average size results from the low-pass filtering effect.

The FOV results obtained in this study still add information about the actual FOV: the retrieved complexity, whether true or not, indicate that there is spatial heterogeneity in the correlation between LR and HR measurements, which cannot be resolved by the correlation technique. This heterogeneity is probably the reason for the observed (tiny) differences in the results of the two compared techniques: (1) the bias between the IFR peak and the FOV disc in Fig. 18b, and (b) the FOV diameter could not unambiguously be retrieved by the correlation technique. Hence, we conclude that the actual FOV had probably the size of the theoretically computed diameter featuring steeper edges, which could not be resolved due to the limited sample size, and that the correlation technique was overestimating the FOV diameter due to the spatial heterogeneities. In summary, however, the influence of the discussed differences on the measured $\mathrm{SO}_{2}$ fluxes are probably minor.

It is noted that IFR is significantly faster than the state-ofthe-art correlation technique, which, however, has not been tuned for speed. Using the same data basis, IFR using LSMR finished after $3.5 \mathrm{~s}$ while the brute-force implementation correlation technique of Lübcke et al. (2013) took as much as $1400 \mathrm{~s}$ to find the best LR/HR correlation within the HRFOV using just one a priori FOV shape. Hence, a speed-up by a factor of 400 could be achieved although less a priori information is required.

\section{Conclusions}

This paper describes IFR, which is an independent method to characterize the effective FOV of instruments with low spatial resolution applying complementary measurements at higher resolution during operation without the need of a dedicated, controlled lab environment. IFR was applied to three instruments: GOME-2 and OMI, which are both satellite instruments, and a ground-based DOAS instrument. The quality of existing FOV parametrizations was assessed using the independently retrieved FOV results.

For GOME-2, spatial aliasing is a known issue that has been ignored in most past studies. The effect is demonstrated using the measurement data themselves and the formulas cor- 
recting for this effect could be verified. Furthermore, specific artefacts caused by unexpected scanner operation could be identified. Future studies of the GOME-2 FOV may be conducted using the nadir-static mode of GOME-2 in which the scanner motor is locked. It should then be possible to retrieve the true IFOV of GOME- 2 because the FOV movement during integration becomes negligible.

For OMI, only approximate solutions could be obtained due to limited correlation caused by the orbital delay of $8 \mathrm{~min}$ between HR and LR measurement. The retrieved FOV distribution is much more complex than for GOME-2. While the FOV of GOME-2 is essentially rectangular, the OMI FOV could be parametrized using a 2-D super-Gaussian FOV model. This FOV model is proposed for future operational use for imaging spectrometers because it is comparatively simple, yet provides sufficient accuracy.

The complexity of the retrieved FOV is expected to inspire more sophisticated treatment of the geolocation of each individual pixel. We see five major applications of more accurate satellite FOV models: (1) correlations between groundbased and satellite measurements, (2) high-resolution mapping of atmospheric pollutants, e.g. $\mathrm{NO}_{2}$ from TROPOMI/S5P, (3) mapping of HR cloud products on the respective LR measurements, (4) retrievals and studies using satellite data incorporating external data sources like albedo and sea-ice maps, and (5) comparisons between satellite measurements and independent data sets like model results. In any case, insufficient knowledge of the FOV or ignoring the spatial aliasing in particular can introduce avoidable errors. It is noted that some of these issues have already been addressed, e.g. the pre-launch instrument calibration of TROPOMI displays a breakthrough for treating the FOV of spectrometers (Leloux, 2016) emphasizing the importance of the topic.

For the $\mathrm{SO}_{2}$ camera, IFR produced comparable results as the frequently used correlation method, but it is at least 2 orders of magnitude faster. IFR is furthermore independent from a priori assumptions about the FOV shape, which renders the method ideal for monitoring the DOAS-FOV shape and position in the field. However, interferences with plume movement and acquisition time differences may introduce artefacts in the result.
IFR is particularly efficient and easy to implement. Reasonable results were obtained also for underdetermined problems, for which, however, an error estimation is more difficult (Yao et al., 1999). The authors hope that this study will inspire $\mathrm{SO}_{2}$-camera operators and instrument developers to use the proposed method in order to monitor their FOV pointing and shape on a regular basis. The same applies to satellite instruments, whose FOV parameters may actually change during operation and which are usually inaccessible for calibration experiments after launch. In another recently published study (Verhoelst et al., 2015) the actual mapping of satellite sensitivity is discussed in great detail. Studies like this, where the focus lays in the comparison to ground-based measurements, significantly depend on the FOV of the satellite, whose actual distribution can now be assessed and monitored during operation more efficiently.

Vice versa, also radiometric calibration of satellite-borne instruments (e.g. McCorkel et al., 2016) will probably benefit from better knowledge of the FOV. In particular, it should be possible to retrieve the spectral response functions of HR instruments from co-located LR measurements (R. Siddans, personal communication, 2015).

Data availability. GOME-2 and AVHRR level $1 \mathrm{~b}$ data are provided by EUMETSAT and can be ordered at http://www.eumetsat. int/website/home/Data/DataDelivery/DataRegistration/index.html (EUMETSAT, 2017). OMI and MODIS data are provided by NASA at http://disc.gsfc.nasa.gov/Aura/data-holdings/OMI (NASA, 2017a) and http://ladsweb.modaps.eosdis.nasa.gov (NASA, 2017b), respectively. Information on access to ECMWF data is available at http://www.ecmwf.int. The raw $\mathrm{SO}_{2}$ camera images and DOAS spectra can be obtained on request from the authors or Nicole Bobrowski (nicole.bobrowski@iup.uni-heidelberg.de). 


\section{Appendix A: Error estimation}

The measurement errors of the input data applied in this study are not known a priori. In case of the standard leastsquares solution, it is, however, still possible to estimate the goodness of the fit result $c$ from the reduced $\chi^{2}$, which is defined as the weighted sum of squared errors divided by the degrees of freedom. Assuming uncorrelated measurement errors and without weighting,

$\chi^{2}=\frac{F}{m-n^{\prime}}$

where

$F=\sum_{i=1}^{m} r_{i}^{2}$

is the variance of the residual $\boldsymbol{r}=\boldsymbol{l}-\mathbf{H} \boldsymbol{c}$, and $m-n^{\prime}=m-$ $(n+1)$ approximates the number of degrees of freedom. The diagonal elements of the variance-covariance matrix

$\Sigma=\chi^{2}\left(\mathbf{H}^{T} \mathbf{H}\right)^{-1}$

are now equal to the squared measurement standard deviations

$\sigma_{j}=\sqrt{\left(\Sigma_{c}\right)_{j, j}}$

for $j=0, \ldots, n$.

However, degradation of both HR and LR instruments may further reduce the correlation within the data and, hence, possibly increase the actual error of $c_{j}$. Therefore, the estimated mean standard deviation $\bar{\sigma}=\overline{\sigma_{j}}$ is compared to the empirical standard deviation $\hat{\sigma}$ in order to test the validity of the assumptions. $\hat{\sigma}$ is the standard deviation of a manually defined subset of the retrieved $c_{j}$ for which $c_{j}=0$ is assumed, i.e. grid points outside the inferred FOV.

For IFR applying the LSMR method, however, the ability to obtain error estimates are limited as pointed out by Yao et al. (1999), because the generalized inverse is not solved explicitly and neither the covariance nor the resolution matrix are provided. Therefore, the error is not estimated in cases where LSMR was applied, i.e. for OMI and the ground-based DOAS.

\section{A1 Example: GOME-2}

Before comparing estimated mean standard deviation $\bar{\sigma}$ and empirical standard deviation $\hat{\sigma}$, a matrix analysis of $\hat{\sigma}$ was performed for GOME-2 pixel 12. The $\hat{\sigma}$ was calculated for all LR/HR combinations using all five LR spectral convolution kernels and both short-wave AVHRR channels (Sect. 2.1.1 and Fig. 2). Furthermore, the analysis was conducted both on all $m=100000$ measurements and after measurements over land were removed in order to avoid interferences with chlorophyll absorption and resulting in $m=61066$. The $\hat{\sigma}$ was calculated from the FOV (a) All data $(m=100000)$



Figure A1. Empirical standard deviation $\hat{\sigma}$ depending on LR spectral convolution kernel, HR channel. The matrix analysis is performed (a) on unfiltered data and (b) on a subset containing only measurements over ocean to avoid interference with chlorophyll absorption. The underlined parameter combinations feature lowest $\hat{\sigma}$ values for the lower $(<700 \mathrm{~nm})$ and the upper $(>700 \mathrm{~nm})$ wavelength range in Fig. 2, respectively.

results outside a rectangular mask potentially containing FOV contributions: values inside $-60 \mathrm{~km}<x<44 \mathrm{~km}$ and $-24 \mathrm{~km}<y<24 \mathrm{~km}$ were discarded.

The results in Fig. A1 show that the lowest empirical standard deviation $\hat{\sigma}$ was achieved using unfiltered LR and HR data applying the $630 \mathrm{~nm}$ LR convolution kernel and AVHRR channel 1, respectively. Apparently, the higher number of measurements outweighs the correlation increase by the ocean filter. However, for GOME-2 measurements at longer wavelengths $>700 \mathrm{~nm}$, where spatial aliasing is reduced, including only measurements over ocean significantly increases the quality of the results only if AVHRR channel 1 is applied but not for AVHRR channel 2. It is noted that applying the filter improved $\hat{\sigma}$ for LR/HR comparisons across the red edge in general as expected: $\hat{\sigma}$ was significantly decreased for shortwave LR versus longwave HR and vice versa (yellow and orange colours in Fig. A1b compared to red colours $\left(\hat{\sigma}>5 \times 10^{3}\right)$ in Fig. A1a).

After the optimal LR/HR combination for GOME-2 was found, the dependence of $\bar{\sigma}$ and $\hat{\sigma}$ on sample number $m$ and ocean filter was investigated. Figure A2 illustrates the results using random selections of the basic populations of all data and data collected over ocean only. For sufficiently large $m \approx 5 \times 10^{3}$, it was observed that (1) the slopes were proportional to $m^{-1 / 2}$ (dashed purple lines), (2) excluding pixels over land improved retrieval noise, and (3) the empirical standard deviations were larger than those inferred from theoretical considerations.

These results lead to the conclusion that the inter-pixel variations in the discrete FOV grid are, in principle, independent and that the theoretical limit has not been reached for $m=10^{5}$. Hence, the noise of IFR results may be further reduced by increasing $m$. There are, however, signs that 


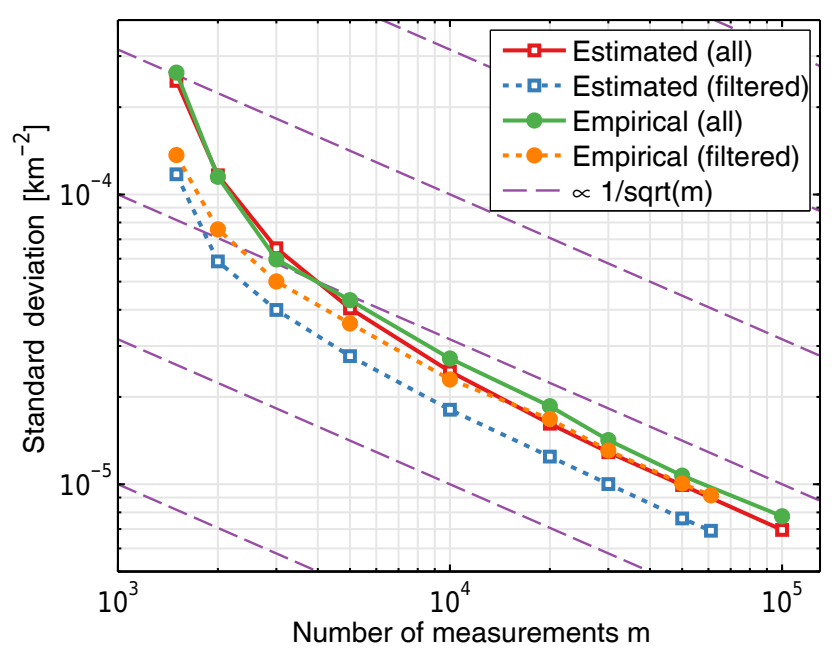

Figure A2. Comparison of estimated mean standard deviation $\bar{\sigma}$ (empty squares) and empirical standard deviation $\hat{\sigma}$ (filled circles) depending on number of measurements $m$ included. The results denoted 'filtered' (dotted lines) are solely based on measurements over ocean. LR convolution kernel is centred at $630 \mathrm{~nm}$ and HR data are from AVHRR channel 1.

there are systematic biases, whose importance may increase at larger $m$. For constant $m$, the retrieval standard deviation decreases if pixels over land are filtered. This indicates that there are differences in the spectral response between HR and LR measurement. The observation that $\bar{\sigma}$ underestimates $\hat{\sigma}$ has at least two possible explanations: (1) $\bar{\sigma}$ does not include all error contributions and (2) there are FOV contributions from outside the FOV mask used for calculating $\hat{\sigma}$. It may be however assumed that $\hat{\sigma}$ is an appropriate error estimator. 


\section{The Supplement related to this article is available online at doi:10.5194/amt-10-881-2017-supplement.}

Competing interests. The authors declare that they have no conflict of interest.

Acknowledgements. Funding by DLR Bonn under grant number 50EE124 is acknowledged. Richard Siddans (RAL) and Katja Grossmann (IUP) are acknowledged for inspiring discussion and Bert van den Oord (KNMI) for technical assistance. Many people were involved in building the $\mathrm{SO}_{2}$ camera prototype; P. Lübcke acknowledges in particular the work of Sebastian Illing and Marco Huwe (both IUP) and is grateful for helpful tips for the design from Denis Pöhler. The $\mathrm{SO}_{2}$-camera measurements were performed in the scope of the IAVCEI 12th Workshop on Volcanic gases. Help during measurements by Nicole Bobrowski, Florian Dinger and Ulrich Platt (all IUP) was gratefully received. GOME-2 and AVHRR level-1 data were provided by EUMETSAT. OMI level-1 data were provided by NASA and downloaded from the Goddard Earth Sciences Data and Information Services Center (GES DISC). MODIS level-1 data were provided by NASA and downloaded from the Level 1 and Atmosphere Archive and Distribution System (LAADS). ECMWF operational data were obtained from the ECMWF Data Server.

The article processing charges for this open-access publication were covered by the Max Planck Society.

Edited by: P. Stammes

Reviewed by: three anonymous referees

\section{References}

Barnes, W., Pagano, T. S., and Salomonson, V.: Prelaunch Characteristics of the Moderate Resolution Imaging Spectroradiometer (MODIS) on EOS-AM1, IEEE Trans. Geosci. Remote Sens., 36, 1088-1100, doi:10.1109/36.700993, 1998.

Bluth, G. J. S., Shannon, J. M., Watson, I. M., Prata, A. J., and Realmuto, V. J.: Development of an ultra-violet digital camera for volcanic $\mathrm{SO}_{2}$ imaging, J. Volcanol. Geoth. Res., 161, 47-56, doi:10.1016/j.jvolgeores.2006.11.004, 2007.

Callies, J., Corpaccioli, E., Eisinger, M., Hahne, A., and Lefebvre, A.: GOME-2 - MetOp's Second-Generation Sensor for Operational Ozone Monitoring, ESA Bulletin, 102, 28-36, 2000.

Campagnolo, M. L. and Montaño, E. L.: Estimation of Effective Resolution for Daily MODIS Gridded Surface Reflectance Products, IEEE Trans. Geosci. Remote Sens., 52, 5622-5632, doi:10.1109/TGRS.2013.2291496, 2014.

Cracknell, A.: The Advanced Very High Resolution Radiometer, Taylor and Francis, London, ISBN 9780748402090, 1997.

de Graaf, M., Sihler, H., Tilstra, L. G., and Stammes, P.: How big is an OMI pixel?, Atmos. Meas. Tech., 9, 3607-3618, doi:10.5194/amt-9-3607-2016, 2016.

Dobber, M. R., Dirksen, R. J., Levelt, P. F., van den Oord, G. H. J., Voors, R., Kleipool, Q., Jaross, G., Kowalewski, M., Hilsenrath, E., Leppelmeier, G., de Vries, J., Dierssen,
W., and Rozemeijer, N.: Ozone Monitoring Instrument calibration, IEEE Trans. Geosci. Remote Sens., 44, 1209-1238, doi:10.1109/TGRS.2006.869987, 2006.

EUMETSAT: AVHRR Level $1 \mathrm{~b}$ Product Guide, Internet, available at: http://www.eumetsat.int/website/wcm/idc/ idcplg?IdcService=GET_FILE\&dDocName=PDF_AVHRR L1B_PRODUCT_GUIDE\&RevisionSelectionMethod= LatestReleased\&Rendition=Web (last access: 20 Oktober 2016), 2011a.

EUMETSAT: GOME-2 FM2-2 Calibration: field of view, doc. MO-TR-TPD-GO-0122-i1.pdf in GOME-2 FM2 key data issue 2 available at: ftp://ftp.eumetsat.int/pub/EPS/out/GOME/ Calibration-Data-Sets/Calibration-Key-Data/FM2-Metop-B/ GOME2-Key-Data-FM2-Issue2.tar.gz (last access: March 2017), 2011b.

EUMETSAT: EPS Product Quality Monitoring Reports - MetOpA: GOME-2, Internet, available at: http://oiswww.eumetsat.int/ epsreports/html/index.php?instrument=GOME\&sat=M02 (last access: 20 November 2015), 2015a.

EUMETSAT: Polar Multi-Sensor Aerosol Product: ATBD, Tech. Rep. EUM/TSS/SPE/14/739904, EUMETSAT, available at: http://www.eumetsat.int/website/wcm/idc/idcplg? IdcService=GET_FILE $\&$ dDocName=PDF_PMAP_ATBD_ PFS\&RevisionSelectionMethod=LatestReleased (last access: March 2017), issue v3B, 2015b.

EUMETSAT: GOME-2 Factsheet, Internet, available at: http: //www.eumetsat.int/ (last access: February 2017), Doc. No. EUM/OPS/DOC/10/1299, Issue v4B, 17 March 2015, 2015 c.

EUMETSAT: GOME-2 and AVHRR data, available at: http://www.eumetsat.int/website/home/Data/DataDelivery/ DataRegistration/index.html, last access: March 2017.

Fong, D. C.-L. and Saunders, M. A.: LSMR: An iterative algorithm for sparse least-squares problems, SIAM J. Sci. Comput., 33, 2950-2971, 2011.

Kern, C., Kick, F., Lübcke, P., Vogel, L., Wöhrbach, M., and Platt, U.: Theoretical description of functionality, applications, and limitations of $\mathrm{SO}_{2}$ cameras for the remote sensing of volcanic plumes, Atmos. Meas. Tech., 3, 733-749, doi:10.5194/amt-3733-2010, 2010.

Kern, C., Lübcke, P., Bobrowski, N., Campion, R., Mori, T., Smekens, J.-F., Stebel, K., Tamburello, G., Burton, M., Platt, U., and Prata, F.: Intercomparison of $\mathrm{SO}_{2}$ camera systems for imaging volcanic gas plumes, J. Volcanol. Geoth. Res., 300, 22-36, doi:10.1016/j.jvolgeores.2014.08.026, 2015a.

Kern, C., Sutton, J., Elias, T., Lee, L., Kamibayashi, K., Antolik, L., and Werner, C.: An automated $\mathrm{SO}_{2}$ camera system for continuous, real-time monitoring of gas emissions from Kīlauea Volcano's summit Overlook Crater, J. Volcanol. Geoth. Res., 300, 81-94, doi:10.1016/j.jvolgeores.2014.12.004, 2015 b.

KNMI: Background information about the Row Anomaly in OMI, available at: http://www.knmi.nl/omi/research/product/ rowanomaly-background.php (last access: 24 February 2016), 2015.

Koelemeijer, R. B. A., Stammes, P., and Watts, P. D.: Comparison of Visible Calibrations of GOME and ATSR-2, Remote Sens. Environ., 63, 279-288, 1998.

Kroon, M., Dobber, M. R., Dirksen, R., Veefkind, J. P., van den Oord, G. H., and Levelt, P. F.: Ozone Monitoring Instru- 
ment geolocation verification, J. Geophys. Res., 113, D15S12, doi:10.1029/2007JD008821, 2008.

Kuhlmann, G., Hartl, A., Cheung, H. M., Lam, Y. F., and Wenig, M. O.: A novel gridding algorithm to create regional trace gas maps from satellite observations, Atmos. Meas. Tech., 7, 451467, doi:10.5194/amt-7-451-2014, 2014.

Kurosu, T. P. and Celarier, E. A.: OMIPIXCOR Readme File, available at: http://disc.sci.gsfc.nasa.gov/Aura/data-holdings/OMI/ documents/v003/OMPIXCOR_README_V003.pdf (last access: August 2014), 2010

Lang, R.: GOME-2 PMD Band Definitions 3.0 and PMD Calibration, EUMETSAT, available at: http://www.eumetsat.int (last access: February 2017), Doc.No. EUM/OPS-EPS/DOC/07/0601, Issue v8, 2010.

Leloux, J.: readme for TROPOMI pixel response function, Memo, KNMI, available at: http://www.tropomi.eu/sites/default/files/ files/S5P-KNMI-OCAL-0150-ME-readme_for_TROPOMI_ pixel_response_function-1.0.0-20160401.pdf (last access: February 2017), issue 1.0.0, 2016.

Levelt, P. F., van den Oord, G. H. J., Dobber, M. R., Malkki, A., Visser, H., de Vries, J., Stammes, P., Lundell, J. O. V., and Saari, H.: The Ozone Monitoring Instrument, IEEE Trans. Geosci. Remote Sens., 44, 1093-1101, doi:10.1109/TGRS.2006.872333, 2006.

Lübcke, P., Bobrowski, N., Illing, S., Kern, C., Alvarez Nieves, J. M., Vogel, L., Zielcke, J., Delgado Granados, H., and Platt, U.: On the absolute calibration of SO2 cameras, Atmos. Meas. Tech., 6, 677-696, doi:10.5194/amt-6-677-2013, 2013.

McCorkel, J., Cairns, B., and Wasilewski, A.: Imager-to-radiometer in-flight cross calibration: RSP radiometric comparison with airborne and satellite sensors, Atmos. Meas. Tech., 9, 955-962, doi:10.5194/amt-9-955-2016, 2016.

McGillem, C. D., Anuta, P. E., Malaret, E., and Yu, K. B.: Estimation of a remote sensing system point-spread function from measured imagery, LARS Technical Reports 81, Purdue University, available at: http://docs.lib.purdue.edu/larstech/81 (last access: February 2017), 1983.

Mori, T. and Burton, M.: The $\mathrm{SO}_{2}$ camera: A simple, fast and cheap method for ground-based imaging of $\mathrm{SO}_{2}$ in volcanic plumes, Geophys. Res. Lett., 33, L24804, doi:10.1029/2006GL027916, 2006.

Munro, R., Eisinger, M., Anderson, C., Callies, J., Corpaccioli, E., Lang, R., Lefebvre, A., Livschitz, Y., and Albiñana, A. P.: GOME-2 on MetOp, in: Proc. of The 2006 EUMETSAT Meteorological Satellite Conference, Helsinki, Finland, 12-16 June 2006, EUMETSAT, p. 48, 2006.

Munro, R., Lang, R., Klaes, D., Poli, G., Retscher, C., Lindstrot, R., Huckle, R., Lacan, A., Grzegorski, M., Holdak, A., Kokhanovsky, A., Livschitz, J., and Eisinger, M.: The GOME2 instrument on the Metop series of satellites: instrument design, calibration, and level 1 data processing - an overview, Atmos. Meas. Tech., 9, 1279-1301, doi:10.5194/amt-9-1279-2016, 2016.

NASA: The Afternoon Constellation, available at: http://atrain.nasa. gov/ (last access: February 2015), 2014.

NASA: OMI data, available at: http://disc.gsfc.nasa.gov/Aura/ data-holdings/OMI, last access: March 2017a.

NASA: MODIS data, available at: http://ladsweb.modaps.eosdis. nasa.gov, last access: March 2017b.
NOAA: NOAA-KLM User's Guide - Section 3.1, available at: http://www.ncdc.noaa.gov/oa/pod-guide/ncdc/docs/klm/ html/c3/sec3-1.htm (last updated 3 March 2009, last access 20 Oktober 2016), 2009.

NOAA: NOAA KLM User's Guide - Appendix D.5, available at: http://www.ncdc.noaa.gov/oa/pod-guide/ncdc/docs/klm/ html/d/app-d5.htm (last updated 13 April 2009, last access 20 Oktober 2016), 2014.

Paige, C. C. and Saunders, M. A.: LSQR: An Algorithm for Sparse Linear Equations And Sparse Least Squares, ACM Trans. Math. Soft., 8, 43-71, 1982.

Platt, U. and Stutz, J.: Differential Optical Absorption Spectroscopy - Principles and Applications, Springer, Berlin, Heidelberg, New York, 1st edn., 2008.

Ruiz, C. P. and Lopez, F. J. A.: Restoring SPOT images using PSFderived deconvolution filters, Int. J. Remote Sensing, 23, 2379 2391, doi:10.1080/01431160110075857, 2002.

Salomonson, V., Barnes, W., Maymon, P. W., Montgomery, H., and Ostrow, H.: MODIS: Advanced Facility Instrument for Studies of the Earth as a System, IEEE Trans. Geosci. Remote Sens., 27, 145-153, doi:10.1109/36.20292, 1989.

Schoeberl, M. R.: The afternoon constellation: a formation of Earth observing systems for the atmosphere and hydrosphere, in: International Geoscience and Remote Sensing Symposium (IGARRS), Toronto, Canada, 24-28 June 2002, vol. 1, pp. 354356, IEEE, doi:10.1109/IGARSS.2002.1025038, 2002.

Schoeberl, M. R., Douglass, A. R., Hilsenrath, E., Bhartia, P. K., Beer, R., Waters, J. W., Gunson, M. R., Froidevaux, L., Gille, J. C., Barnett, J. J., Levelt, P., and DeCola, P.: Overview of the EOS aura mission, IEEE Trans. Geo. Rem. Sens., 44, 10661074, doi:10.1109/TGRS.2005.861950, 2006.

Siddans, R.: S5P-NPP Cloud Processor, ATBD, RAL Space, available at: http://www.tropomi.eu/sites/default/files/ files/S5P-NPPC-RAL-ATBD-0001_NPP-Clouds_v1p0p0_ 20160212.pdf (last access: February 2017), issue 1.0.0, 2016.

Smekens, J.-F., Clarke, A. B., Burton, M. R., Harijoko, A., and Wibowo, H. E.: $\mathrm{SO}_{2}$ emissions at Semeru volcano, Indonesia: Characterization and quantification of persistent and periodic explosive activity, J. Volcanol. Geoth. Res., 300, 121-128, doi:10.1016/j.jvolgeores.2015.01.006, 2015.

te Plate, M. B., Draaisma, F., de Vries, J., and van den Oord, G. H. J.: Ozone Monitoring Instrument: system description and test results, in: Proc. SPIE 4540 - Sensors, Systems, and NextGeneration Satellites V, edited by: Fujisada, H., Lurie, J. B., and Weber, K., p. 122, doi:10.1117/12.450654, 2001.

Tilstra, L. G., Tuinder, O. N. E., and Stammes, P.: GOME-2 PMD band reflectances - verification report, KNMI Report KNMI-RP-2011-01, available at: http://temis.nl/surface/gome2_ ler/KNMI-RP-2011-01.pdf (last access: February 2017), 2011.

Verhoelst, T., Granville, J., Hendrick, F., Kühler, U., Lerot, C., Pommereau, J.-P., Redondas, A., Van Roozendael, M., and Lambert, J.-C.: Metrology of ground-based satellite validation: co-location mismatch and smoothing issues of total ozone comparisons, Atmos. Meas. Tech., 8, 5039-5062, doi:10.5194/amt-8-5039-2015, 2015.

Wang, Z., Xiong, X., Choi, T., and Link, D.: On-Orbit Characterization of MODIS Modulation Transfer Function Using the Moon, IEEE Trans. Geo. Rem. Sens., 52, 4112-4121, doi:10.1109/TGRS.2013.2279610, 2014. 
Wolfe, R. E., Lin, G., Nishihama, M., Tewari, K. P., Tilton, J. C., and Isaacman, A. R.: Suomi NPP VIIRS prelaunch and on-orbit geometric calibration and characterization, J. Geophys. Res.-Atmos., 118, 11508-11521, doi:10.1002/jgrd.50873, 2013.

Xiong, X., Che, N., and Barnes, W.: Terra MODIS On-Orbit Spatial Characterization and Performance, IEEE Trans. Geosci. Remote Sens., 43, 355-365, doi:10.1109/TGRS.2004.840643, 2005.

Xiong, X., Che, N., and Barnes, W.: Terra MODIS On-Orbit Spectral Characterization and Performance, IEEE Trans. Geosci. Remote Sens., 44, 2198-2206, doi:10.1109/TGRS.2006.872083, 2006.
Xiong, X., Sun, J., Che, N., Angal, A., and Choi, J.: Comparison of Terra and Aqua MODIS VIS Bands On-Orbit Response, in: International Geoscience and Remote Sensing Symposium (IGARSS), Boston, USA, 7-11 July 2008, vol. 4, 1348-1351, IEEE, doi:10.1109/IGARSS.2008.4779981, 2008

Yao, Z. S., Roberts, R. G., and Tryggvason, A.: Calculating resolution and covariance matrices for seismic tomography with the LSQR method, Geophys. J. Int., 138, 886-894, 1999. 